\title{
Ionization of pyridine: interplay of orbital relaxation and electron correlation
}

\author{
A. B. Trofimov, ${ }^{1,2, a)}$ D. M. P. Holland,${ }^{3}$ I. Powis, ${ }^{4}$ R. C. Menzies, ${ }^{4}$ A. W. Potts, ${ }^{5}$ L. \\ Karlsson, ${ }^{6}$ E. V. Gromov, ${ }^{7}$ I. L. Badsyuk, ${ }^{2}$ and J. Schirmer ${ }^{8}$ \\ ${ }^{1}$ Laboratory of Quantum Chemistry, Irkutsk State University, Karl Marx Str. 1, 664003 \\ Irkutsk, Russia \\ ${ }^{2}$ Favorsky's Institute of Chemistry, SB RAS, Favorsky Str. 1, 664033 Irkutsk, Russia \\ ${ }^{3}$ Daresbury Laboratory, Daresbury, Warrington, Cheshire WA4 4AD, United Kingdom \\ ${ }^{4}$ School of Chemistry, University of Nottingham, Nottingham NG7 2RD, United Kingdom \\ ${ }^{5}$ Department of Physics, King's College, Strand, London WC2R 2LS, United Kingdom \\ ${ }^{6}$ Department of Physics and Astronomy, Uppsala University, Box 516, SE-751 20 Uppsala, \\ Sweden \\ ${ }^{7}$ Max Planck Institute for Medical Research, Jahnstraße 29, 69120 Heidelberg, Germany \\ ${ }^{8}$ Theoretische Chemie, Physikalisch-Chemisches Institut, Universität Heidelberg, Im \\ Neuenheimer Feld 229, 69120 Heidelberg, Germany
}

\section{Abstract}

The valence shell ionization spectrum of pyridine was studied using the third-order algebraicdiagrammatic construction $[\mathrm{ADC}(3)]$ approximation scheme for the one-particle Green's function and the outer-valence Green's function (OVGF) method. The results were used to interpret angle resolved photoelectron spectra recorded with synchrotron radiation in the photon energy range of $17-120 \mathrm{eV}$. The lowest four states of the pyridine radical cation, namely ${ }^{2} \mathrm{~A}_{2}\left(1 \mathrm{a}_{2}{ }^{-1}\right),{ }^{2} \mathrm{~A}_{1}\left(7 \mathrm{a}_{1}^{-1}\right),{ }^{2} \mathrm{~B}_{1}\left(2 \mathrm{~b}_{1}^{-1}\right),{ }^{2} \mathrm{~B}_{2}\left(5 \mathrm{~b}_{2}{ }^{-1}\right)$, were studied in detail using various high-level electronic structure calculation methods. The vertical ionization energies were established using the equation-of-motion coupled-cluster approach with single, double and triple excitations (EOM-IP-CCSDT) and the complete basis set (CBS) extrapolation technique. Further interpretation of the electronic structure results was accomplished using Dyson orbitals, electron density difference plots, and a second-order perturbation theory treatment for the relaxation energy. Strong orbital relaxation and electron correlation effects were shown to accompany ionization of the $7 a_{1}$ orbital, which formally represents the nonbonding $\sigma$-type nitrogen lone-pair $(n \sigma)$ orbital. The theoretical work establishes the important roles of the $\pi$-system ( $\pi-\pi^{*}$ excitations) in the screening of the no-hole and of the

\footnotetext{
a) Email: atrof@math.isu.runnet.ru, abtrof@mail.ru
} 

parameters were computed using the MP2 (second-order Møller-Plesset perturbation theory) and CCSD (coupled-cluster singles and doubles) methods, and the harmonic vibrational frequencies were obtained at the MP2 level of theory for the lowest three cation states. The results were used to estimate the adiabatic 0-0 ionization energies, which were then compared to the available experimental and theoretical data. Photoelectron anisotropy parameters and photoionization partial cross-sections, derived from the experimental spectra, were compared to predictions obtained with the continuum multiple scattering approach. 


\section{INTRODUCTION}

Pyridine (Figure 1) is an important heterocyclic molecule, and is closely related to benzene and more complex six-membered nitrogen-containing aromatic molecules. Such molecules occur as structural units in many biological compounds. Hence, an understanding of the electronic structure of pyridine is essential to many fields of organic chemistry including biochemistry, medicinal and pharmaceutical chemistry. ${ }^{1,2}$

Photoelectron spectroscopy, especially when carried out with a continuously tunable photon source, provides an ideal means of exploring molecular electronic structure. The results obtained from such experiments can be interpreted with the help of quantum chemical calculations. The latter can be performed using various approximate computational schemes ranging from Hartree-Fock (HF) theory (Koopmans' theorem) to highly sophisticated methods aiming to eliminate shortcomings of the one-electron approximation. ${ }^{3}$ The improvements with respect to the Koopmans level of theory are usually discussed in terms of two effects, namely orbital relaxation and electron correlation. Whereas orbital relaxation (i.e. the response of the electronic structure to a hole created in the occupied orbitals) can be accounted for using HF calculations performed separately for the initial and final states [the so-called delta selfconsistent field $(\triangle \mathrm{SCF})$ approach], electron correlation can be treated only through methods going beyond the HF level of theory.

Orbital relaxation and electron correlation are treated consistently in the Green's function (electron propagator) methods. ${ }^{3,4}$ Here, computational schemes within the algebraicdiagrammatic construction (ADC) approach $^{5-11}$ have proved to be very useful, $4,7,8,11-13$ especially the third-order ADC schemes [ADC(3)] using the Dyson equation, ${ }^{5-8}$ or a more direct non-Dyson framework. ${ }^{9-11}$ Results of a similar quality can be obtained in many cases using the closely related two-particle-one-hole Tamm-Dancoff, ${ }^{7} 3+,{ }^{14}$ and nondiagonal renormalized second-order ${ }^{15}$ methods, as well as the simpler outer-valence Green's function $(\mathrm{OVGF})^{7,14,16}$ and partial third-order quasiparticle ${ }^{17,18}$ diagonal self-energy methods.

Reliable results for ionization potentials (IPs) can also be obtained within the framework of the equation-of-motion (EOM) coupled-cluster $(\mathrm{CC})$ theory (EOM-IP-CC) $)^{19-21}$ at the level of singles and doubles (CCSD) and higher CC models, ${ }^{22}$ or by using the 
theoretically equivalent symmetry-adapted cluster configuration interaction (SAC-CI) method. ${ }^{23}$ Accurate predictions of ionization energies for the lowest cationic state in each spatial symmetry are possible when separate $\mathrm{CC}$ calculations for the neutral and cationic species are performed ( $\triangle \mathrm{CC}$ approach).

The methods mentioned above normally yield consistent results, suitable for the unambiguous interpretation of valence-shell photoelectron spectra, except for certain difficult cases where the theoretical predictions diverge. One such example concerns the two lowest states, ${ }^{2} \mathrm{~A}_{1}\left(\mathrm{n} \sigma^{-1}\right)$ and ${ }^{2} \mathrm{~A}_{2}\left(\pi^{-1}\right)$, of the pyridine cation. These states are close in energy, and their vertical ordering has not been established conclusively either experimentally ${ }^{24-39}$ or theoretically, ${ }^{35,40-48}$ although there is a general consensus, based on experimental evidence, that the ${ }^{2} \mathrm{~A}_{1}\left(n \sigma^{-1}\right)$ state is at least adiabatically below the ${ }^{2} \mathrm{~A}_{2}\left(\pi^{-1}\right)$ state. The calculated ionization energy of the ${ }^{2} \mathrm{~A}_{1}\left(n \sigma^{-1}\right)$ state varies considerably, depending on the theoretical treatment, whereas the results for the ${ }^{2} \mathrm{~A}_{2}\left(\pi^{-1}\right)$ state are more stable.

Similar effects concerning nearly degenerate energy levels, due to the ionization of nonbonding n $\sigma$-type lone-pairs (LPS) and $\pi$-orbitals, has been observed in other heteroaromatic molecules, such as adenine, ${ }^{49-51}$ pyrimidine ${ }^{52}$ and purine, ${ }^{52}$ where the LPs belong to the nitrogen atoms. In thymine, ${ }^{50,51,53}$ uracil, and the uracil derivatives, ${ }^{54-56}$ the LPs involved belong to the oxygen atoms outside the aromatic ring; and in guanine ${ }^{51,57,58}$ the LPs have a mixed nitrogen-oxygen character.

Another important issue related to the theoretical treatment of the nearly-degenerate no$\pi$ pairs is that the no- and $\pi$-states are initially always strongly separated at the HF (Koopmans' theorem) level of theory, but merge together when a more accurate treatment is employed. The shifts in the HF ionization energies are non-uniform, and are much larger for the n $\sigma$-leyels than for the $\pi$-levels. This effect is more pronounced in the propagator results ${ }^{35,47,48,50,53-59}$ than in those employing the EOM-IP-CC approach. ${ }^{51}$

Previously, the ionization of pyridine has been studied theoretically by Suzuki and Suzuki, ${ }^{41}$ using the continuum multiple scattering $\mathrm{X} \alpha(\mathrm{CMS}-\mathrm{X} \alpha)$ method, by Wan et al., ${ }^{42}$ and Kitao and Nakatsuji, ${ }^{43}$ using the SAC-CI method, and by Plashkevych et al. ${ }^{44}$ using Kohn-Sham density functional and transition potential methodology. Yang et al. ${ }^{45}$ used the 
direct static exchange approach, which takes cross-section effects into account, to investigate the photoelectron spectra of pyridine and some of its derivatives. Lorentzon et al. ${ }^{46}$ computed the lowest ionization potentials using second-order complete-active space perturbation theory (CASPT2). Walker et al. ${ }^{40}$ studied a large number of cationic states spanning the entire outervalence energy domain using multi-reference configuration interaction methods with singly and doubly excited configurations (MRD-CI). The ionization spectrum of pyridine has also been treated using many-body Green's function techniques. ${ }^{35,47,48}$

A comprehensive review of the corresponding experimental work, carried out prior to 1988 , has been provided by Innes et al. ${ }^{24}$ Many of the early studies focused on identifying the molecular orbitals giving rise to the first two bands in the photoelectron spectrum. The outermost band in the HeI excited photoelectron spectrum, ${ }^{25-30}$ observed at binding energies between 9.2 and $10.2 \mathrm{eV}$, is predicted to correspond to ionization from the $7 \mathrm{a}_{1}\left(\sigma_{\mathrm{N} L P}\right)$ and $1 \mathrm{a}_{2}(\pi)$ orbitals. This band is partially overlapped by the second band, centred around $10.5 \mathrm{eV}$ and due to the $2 b_{1}(\pi)$ orbital ionization. Band shape considerations led Gleiter et al. ${ }^{26}$ to associate the $7 \mathrm{a}_{1}$ and $1 \mathrm{a}_{2}$ orbitals with the highest occupied molecular orbital (HOMO) and HOMO-1, respectively, although King et al., ${ }^{27}$ based upon fluoro-substituent effects, preferred the reverse ordering.

Another experimental approach which is often useful in assigning a particular molecular orbital to a specific photoelectron band involves measuring the photoelectron angular distributions. This technique was used in the HeI excited photoelectron study performed by Utsunomiya et al. ${ }^{29}$ to determine the anisotropy parameters, as a function of binding energy, of the outermost bands of pyridine and dimethylpyridine. For the dimethyl substituted molecule, the molecular orbital sequence is well established. A comparison between the anisotropy parameters for dimethylpyridine and those for pyridine resulted in Utsunomiya et al. assigning the lowest ionization in pyridine to the $7 \mathrm{a}_{1}$ orbital.

Photoelectron angular distribution experiments have also been performed by Piancastelli et al. ${ }^{31}$ employing synchrotron radiation. Their measurements showed that the anisotropy parameter for the high binding energy region of the first band increased more rapidly as the photon energy increased from 13 to $27 \mathrm{eV}$ than did the anisotropy parameter for 
the low binding energy region. Since a rapid increase in the value of the anisotropy parameter is typical for electron ejection from a $\pi$-orbital, Piancastelli et al. ${ }^{31}$ associated the low binding energy region of the first band predominantly to contributions from the $7 a_{1}\left(\sigma_{N L P}\right)$ orbital. Similar conclusions were reached by Berg et al. ${ }^{32}$ using multiphoton ionization.

Subsequent experimental studies include photoelectron spectra recorded with $\mathrm{HeI}^{33,34}$ or synchrotron $^{35,36}$ radiation, a resonance-enhanced multiphoton ionization study, ${ }^{37}$ and mass analyzed threshold ionization (MATI) investigations. ${ }^{38,39}$ The emphasis of these recent studies lay in obtaining an improved understanding of the spectroscopic properties of the pyridine cation. In contrast, the valence shell photoionization dynamics have not been investigated in a detailed manner, either experimentally or theoretically.

The results from earlier calculations have allowed many of the bands observed in the photoelectron spectrum to be assigned but, as already mentioned, inconsistencies remain with respect to the position of the ${ }^{2} \mathrm{~A}_{1}\left(\mathrm{n} \sigma^{-1}\right)$ state and of some of the other states. The difficulty concerning the ${ }^{2} \mathrm{~A}_{1}\left(\mathrm{n} \sigma^{-1}\right)$ state is related to a more general issue regarding the ionization of no-orbitals in heteroatomic molecules. The present work addresses this issue by analyzing the lowest $\sigma$ - and $\pi$-type cationic states of pyridine in terms of orbital relaxation and electron correlation effects. We use the ADC(3), OVGF and EOM-IP-CC methods and present an analysis of relaxation energies based on second-order perturbation theory, together with electron density difference plots, and Dyson molecular orbitals. We also measure valence shell photoelectron angular distributions and photoionization partial cross-sections, and compare these experimental results to theoretical predictions obtained with the CMS- X $\alpha$ approach, to help characterize the photoionization dynamics.

\section{THEORY AND COMPUTATIONAL DETAILS OF ELECTRONIC STRUCTURE CALCULATIONS}

The computation of the energies and photoelectron intensities of the outer- and inner-

valence vertical ionization transitions of pyridine was based on the ab initio Green's function (GF) electronic structure methods as used in our previous studies of halogenated benzenes ${ }^{11,12}$ and other heterocyclic molecules..$^{50,53-55,57}$ More specifically, the ADC(3) scheme for the one- 
Publishing particle Green's function ${ }^{5-8}$ was employed. For the outer valence orbitals the less rigorous but more economic OVGF method ${ }^{7,14}$ was also used. The results of the calculations were analyzed with the aid of Dyson molecular orbitals for the lowest four ionization transitions. The electron density difference maps for HF and coupled-cluster doubles (CCD) densities were plotted, and the second-order perturbation-theoretical treatment of the relaxation energy was employed to obtain a better insight into the electron correlation and orbital relaxation effects in the ionization of these orbitals.

\section{A. Third-order algebraic-diagrammatic construction approach to the one- particle Green's function}

In the $\mathrm{ADC}(3)$ scheme for the one-particle Green's function, ${ }^{5-8}$ the vertical ionization energies and the corresponding transition probabilities are obtained as the solutions of the Hermitian eigenvalue problem:

$$
\mathbf{B X}=\mathbf{X E}, \quad \mathbf{X}^{\dagger} \mathbf{X}=\mathbf{1},
$$

where the matrix $\mathbf{B}$ is defined according to:

$$
\mathbf{B}=\left(\begin{array}{ccc}
\boldsymbol{\varepsilon}+\boldsymbol{\Sigma}(\infty) & \left(\mathbf{U}^{+}\right) & \left(\mathbf{U}^{-}\right) \\
\mathbf{U}^{+} & (\mathbf{K}+\mathbf{C})^{+} & 0 \\
\mathbf{U}^{-} & 0 & (\mathbf{K}+\mathbf{C})^{-}
\end{array}\right)
$$

Here $\boldsymbol{\varepsilon}$ is the diagonal matrix of the HF orbital energies, and $\boldsymbol{\Sigma}(\infty)$ is the constant part of the self-energy. Both quantities are defined with respect to the space of one-hole $(1 h)$ and oneparticle (1p) configurations with respect to the HF ground state. The matrices $(\mathbf{K}+\mathbf{C})^{ \pm}$and $\mathbf{U}^{ \pm}$are referred to as matrices of effective interactions and effective coupling amplitudes, respectively. Their definition involves configuration spaces of two hole-one particle $(2 h-1 p)$ and two particle-one hole $(2 p-1 h)$ configurations, indicated by "minus" and "plus" superscripts, respectively. The elements of the $\boldsymbol{\Sigma}(\infty),(\mathbf{K}+\mathbf{C})^{ \pm}$, and $\mathbf{U}^{ \pm}$matrices have the form of the finite perturbation theoretical (PT) expansions through third order with respect to the residual electron interaction beyond the HF picture. In the present version of the ADC(3) method, the constant part $\Sigma(\infty)$ is treated at least through fourth order according to the procedure described in Ref. 6, which significantly improves the quality of the results. The 
specific form of the matrix $\mathbf{B}$ originates from the treatment of the Dyson equation in which the ADC representation of the dynamic self-energy part, $\Sigma(\omega)$, is used. The fact that the $(N-1)$ - and $(N+1)$-electron spaces are coupled is a consequence of the Dyson equation connecting respective parts of the Green's function. ${ }^{3,59,60}$

The eigenvalues $e_{n}\left(E_{n m}=e_{n} \delta_{m n}\right)$ of Eq. (1) are the negative vertical ionization energies $e_{n}=-\left(E_{n}^{N-1}-E_{0}\right)$ and electron affinities $e_{n}=-\left(E_{0}-E_{n}^{N+1}\right)$ of the system under consideration. They are the poles of the one-particle Green's function in its spectroscopic representation. The corresponding residues are defined as $x_{p}^{(n)} x_{q}^{(n)^{*}}$ products of the eigenvector components $x_{p}^{(n)}=X_{p n}$. The quantities $x_{p}^{(n)}$, also referred to as spectroscopic factors, define, for ionization, the probability of finding the final ionic state $\left|\Psi_{n}^{N-1}\right\rangle$ in the form of a pseudo-state $a_{p}\left|\Psi_{0}^{N}\right\rangle$, produced by the sudden ejection of an electron out of the molecular orbital $\left|\varphi_{p}\right\rangle$ in the initial state $\left|\Psi_{0}^{N}\right\rangle$ (here $a_{p}$ is the appropriate destruction operator):

$$
x_{p}^{(n)}=\left\langle\Psi_{n}^{N-1}\left|a_{p}\right| \Psi_{0}^{N}\right\rangle .
$$

The spectroscopic factors $x_{p}^{(n)}$ (also known as Feynman-Dyson amplitudes) allow one to evaluate the "pole strength" $P_{n}$, which is used as an approximate measure of the relative spectral intensity:

$$
P_{n}=\sum_{p}\left|x_{p}^{(n)}\right|^{2}
$$

where the summation is performed over the whole range of molecular orbitals (MOs).

In the ADC(3) method, both $e_{n}$ and $x_{p}^{(n)}$ are treated consistently through third order of PT with respect to the main photoelectron transitions (producing cationic states of $1 h$-type). The photoelectron satellite states characterized as $2 h-1 p$ excitations are treated consistently only through first order, so that a less accurate description is afforded in spectral regions dominated by such transitions. Nevertheless, the ADC(3) method allows a fairly reliable description of cationic states characterized as strong $1 h / 2 h-1 p$ mixtures. Such mixtures are often encountered at higher ionization energies where a "break-down of the one-electron picture of ionization" may occur. ${ }^{4}$ This capability is not only important for an adequate 
description of the inner-valence region of the ionization spectra, but also for the treatment of heterocyclic molecules (which includes pyridine) where an early onset of the "selective" break-down phenomenon, characterized by the strong redistribution of intensity from the inner $\pi$-type MOs to various satellites, has been observed. ${ }^{61,62}$

In general, the $\mathrm{ADC}(3)$ method takes into account all physical effects, such as electron correlation in the initial and final states, and orbital relaxation, relevant to ionization. The computational procedure of the $\operatorname{ADC}(3)$ scheme is size-consistent, ${ }^{63}$ and can be implemented as an $n^{5}$ scaling of the computational effort, where $n$ is the number of molecular orbitals (disregarding here the improved fourth-order treatment of $\Sigma(\infty)$ ). This represents one of the best trade-offs between computational effort and theoretical accuracy available today.

\section{B. Dyson orbitals}

The Dyson orbitals (DOs) $\phi_{n}\left(\mathbf{r}_{1}\right)$ are defined ${ }^{64,65}$ as overlaps of the initial $N$-electron ground-state wavefunction $\left|\Psi_{0}^{N}\right\rangle$ and the final $(N-1)$-electron wavefunction $\left|\Psi_{n}^{N-1}\right\rangle$ for the $n$-th state of the cation:

$$
\phi_{n}\left(\mathbf{x}_{1}\right)=(N)^{\frac{1}{2}} \int \Psi_{0}^{N}\left(\mathbf{x}_{1}, \ldots, \mathbf{x}_{N}\right) \Psi_{n}^{N-1}\left(\mathbf{x}_{2}, \ldots, \mathbf{x}_{N}\right)^{*} \mathbf{d} \mathbf{x}_{2} \ldots \mathbf{d} \mathbf{x}_{N},
$$

where the integration is performed over the space-spin coordinates $\mathbf{x}_{i}$ of $(N-1)$ electrons. Eq. (5) can be rewritten using the second-quantization formalism as follows:

$$
\left|\phi_{n}\right\rangle=\sum_{p}\left|\varphi_{p}\right\rangle\left\langle\Psi_{n}^{N-1}\left|c_{p}\right| \Psi_{0}^{N}\right\rangle=\sum_{p} x_{p}^{(n)}\left|\varphi_{p}\right\rangle,
$$

where the summation is performed over all available HF orbitals $\left|\varphi_{p}\right\rangle$. The expansion coefficients $x_{p}^{(n)}$, readily identified as the spectroscopic factors (Eq. (3)) of the one-particle Green's function, are obtained from the $\operatorname{ADC}(3)$ calculations.

The DOs reflect the change in the electronic structure effected by the removal of an electron from the molecule. From Eq. (6) it is also clear that in the HF approximation (where $\left.x_{p}^{(n)}=\delta_{p n}\right)$, the DOs $\left|\phi_{p}\right\rangle$ coincide with the canonical HF orbitals $\left|\phi_{p}\right\rangle \equiv\left|\varphi_{p}\right\rangle$. For correlated methods, the DOs are linear combinations of the HF orbitals; usually there is a single dominant term when there are no closely lying interacting cationic states, and the one-electron picture of ionization holds. 
The following important relation can be obtained for DOs:

$$
\left\langle\phi_{n} \mid \phi_{n}\right\rangle=\sum_{p}\left|x_{p}^{(n)}\right|^{2}=P_{n}
$$

This implies that the norm of $\left|\phi_{n}\right\rangle$ is given by the pole strength $P_{n}$ of the corresponding transition. According to Eq. (3), the spectroscopic factors, and thus the pole strengths, decrease when the $2 h-1 p$ character of $\left|\Psi_{n}^{N-1}\right\rangle$ increases, as is the case when the one-electron picture of ionization breaks-down. ${ }^{4}$ In such a situation, the DOs are no longer appropriate for the analysis of changes in the electronic structure upon ionization. Even if $2 h-1 p$ admixtures do not compromise the one-electron picture of ionization, the respective changes are hardly reflected by the DOs. This shows the limitations of the DOs with respect to e.g. such an important effect as orbital relaxation, manifesting itself by $2 h-1 p$ admixtures in the final wave function. ${ }^{5,66}$

The Dyson orbitals can be used for studies of various ionization related phenomena, ${ }^{67-}$ ${ }^{71}$ as well as for the visualization of the results of the Green's function calculations. ${ }^{49,53}$ In the present study we employ Dyson orbitals to analyze the ADC(3) results for pyridine.

\section{The electron density and electron density differences}

Another option to analyze the changes in the electronic structure upon ionization is to consider the many-electron density differences. The initial $\mathrm{N}$-electron ground-state density function $\rho^{N}\left(\mathbf{r}_{1}\right)$ is defined according to the well-known expression: ${ }^{72}$

$$
\rho^{N}\left(\mathbf{r}_{1}\right)=N \sum_{s_{1} \ldots s_{N}} \int\left|\Psi_{0}^{N}\left(\mathbf{r}_{1}, s_{1}, \mathbf{r}_{2}, s_{2}, \ldots, \mathbf{r}_{N} s_{N}\right)\right|^{2} \mathbf{d r}_{2} \ldots \mathbf{d r}_{N}
$$

where $\left\langle\Psi_{0}^{N}\right\rangle$ as earlier is the ground-state wavefunction; the integration is performed over the space coordinates $\mathbf{r}_{i}$ of $(N-1)$ electrons and the summation is performed over all spin coordinates $s_{i}$. Analogously, the final-state $(N-1)$-electronic density can be written as:

$$
\rho_{n}^{N-1}\left(\mathbf{r}_{1}\right)=(N-1) \sum_{s_{1} \ldots s_{N-1}} \int\left|\Psi_{n}^{N-1}\left(\mathbf{r}_{1}, s_{1}, \mathbf{r}_{2}, s_{2}, \ldots, \mathbf{r}_{N-1} s_{N-1}\right)\right|^{2} \mathbf{d r}_{2} \ldots \mathbf{d r}_{N-1},
$$

where $\left|\Psi_{n}^{N-1}\right\rangle$ is the wavefunction for the cationic state under consideration and the integration is performed over the coordinates of $(N-2)$ electrons.

The density difference function $\Delta \rho_{n}^{N-1 / N}$, defined as 


$$
\Delta \rho_{n}^{N-1 / N}(\mathbf{r})=\rho_{n}^{N-1}(\mathbf{r})-\rho_{0}^{N}(\mathbf{r})
$$

shows the changes in the electron density upon ionization. In contrast to the Dyson orbitals, $\Delta \rho_{n}^{N-1 / N}$ is not affected by the limitations with respect to $2 h-1 p$ admixtures, and correlated wave functions of arbitrary complexity can be studied. In the present work, we inspect $\Delta \rho_{n}^{N-1 / N}$ to qualitatively investigate orbital relaxation and electron correlation effects in pyridine, using densities computed at the CCD level of theory.

The electron density differences provide a possibility of comparing different theoretical approximations. By comparing the cationic electron densities computed using the HF and $\Delta \mathrm{SCF}$ approaches the changes due to the orbital relaxation, $\Delta \rho_{n}^{N-1(\mathrm{rel})}(\mathbf{r})$, can be isolated and analyzed:

$$
\Delta \rho_{n}^{N-1(\mathrm{rel})}(\mathbf{r})=\rho_{n}^{N-1(\Delta \mathrm{SCF})}(\mathbf{r})-\rho_{n}^{N-1(\mathrm{HF})}(\mathbf{r}) .
$$

Similarly, when the $\triangle \mathrm{SCF}$ electron density for a cationic state is compared to the density computed using the $\mathrm{CCD}$ approach based on relaxed $\triangle \mathrm{SCF}$ orbitals (within the unrestricted HF framework), a density difference function of the form

$$
\Delta \rho_{n}^{N-1(\mathrm{cor})}(\mathbf{r})=\rho_{n}^{N-1(\mathrm{CCD})}(\mathbf{r})-\rho_{n}^{N-1(\Delta \mathrm{SCF})}(\mathbf{r})
$$

allows for an insight into the role of the electron correlation effects.

To display $\Delta \rho(\mathbf{r})$, which depends on three spatial coordinates, we use two-dimensional contour plots (where two/coordinates are varied and one coordinate remains fixed). As sections we chose the plane of the pyridine ring and the parallel plane $0.5 \AA$ above the molecular plane.

\section{Second-order perturbation theory for the relaxation energy}

As mentioned above, the $\triangle \mathrm{SCF}$ approach allows one to fully recover the relaxation energy. The $\triangle \mathrm{SCF}$ results can, in turn, be analyzed by perturbation theory (see Refs. $[66,73]$ and references cited therein), providing useful insight into the nature of the relaxation processes. 
The starting point here is the assumption that the $\triangle \mathrm{SCF}$ result for the IP of the $k$-th orbital can be expressed as the Koopmans' result (neutral ground-state HF orbital energy $\varepsilon_{k}$ taken with the opposite sign) corrected by subtraction of the relaxation energy $\Delta E_{k}^{R}: 66$

$$
\mathrm{IP}_{k}(\Delta \mathrm{SCF})=-\varepsilon_{k}-\Delta E_{k}^{R}
$$

The relaxation energy $\Delta E_{k}^{R}$ for the $k$-th orbital through second order is equal to one half of the screening energy $\Delta E^{S}(k)$ for that orbital: ${ }^{66}$

$$
\Delta E_{k}^{R}=\frac{1}{2} \Delta E^{S}(k)+O(3) \text {. }
$$

Here and further on $O(3)$ denotes the remainder of perturbation series beyond second order.

The screening energy $\Delta E^{S}(k)$ can be expressed as the diagonal matrix element of the so-called screening operator $\hat{\Delta} \rho_{k}$ defined with respect to the $k$-th orbital. ${ }^{66}$ The perturbation expansion for $\Delta E^{S}(k)$ through second order reads:

$$
\Delta E^{S}(k)=\left\langle k\left|\hat{\Delta} \rho_{k}\right| k\right\rangle=2 \sum_{i \neq k, a}(2(k k \mid a i)-(k i \mid a k)) \frac{(k k \mid a i)}{\left(\varepsilon_{a}-\varepsilon_{i}\right)}+O(3) .
$$

Here, $i$ and $a$ denote occupied and unoccupied orbitals, respectively, in the HF ground-state. The Coulomb two-electron matrix elements $(p q \mid r s)$ are given in the " 1122 " notation, and $\varepsilon_{p}$ denote the HF orbital energies. Here and further on in this section the expressions are given in the spin-free form where the summation over spin variables has been performed so that only spatial orbitals come into play.

For further use it can be convenient to rewrite the relaxation energy as follows:

$$
\Delta E_{k}^{R}=\sum_{i \neq k, a} S(k, i a)+O(3)
$$

In Eq. (16) each term $S(k, i a)$ consists of the two parts:

$$
S(k, i a)=S_{1}(k, i a)-S_{2}(k, i a),
$$

where

$$
S_{1}(k, i a)=2 \frac{(k k \mid a i)^{2}}{\left(\varepsilon_{a}-\varepsilon_{i}\right)},
$$

and

$$
S_{2}(k, i a)=\frac{(k k \mid a i)(k i \mid a k)}{\left(\varepsilon_{a}-\varepsilon_{i}\right)} .
$$


Publishing Here we also note that the matrix element $(k k \mid a i)$ can be considered as the matrix element $\left\langle i\left|\hat{J}_{k}\right| a\right\rangle$ of the familiar Coulomb operator $\hat{J}_{k}$ for orbital $k$.

\section{E. Details of the computations}

The ADC(3) calculations were performed using the original code ${ }^{74}$ linked to the Gamess ab initio program package. ${ }^{75}$ The cc-pVDZ basis set ${ }^{76}$ with Cartesian representation of the $d$-functions was employed in the calculations of the spectral profiles. Additionally, the low-lying ionization transitions were computed at the ADC(3) level using the cc-pVTZ basis set. ${ }^{76}$ Block-Davidson diagonalization techniques were used in the evaluation of the most prominent transitions at lower binding energies. The block-Lanczos procedure was applied in the generation of the entire spectral envelope, thereby allowing low-intensity satellite contributions to be taken into account. The theoretical spectral profiles were constructed by convoluting the ADC(3) energies with Lorentzians of $0.4 \mathrm{eV}$ FWHM (full width at half maximum).

The ground-state geometrical parameters of pyridine were obtained through a full geometry optimization using MP2 theory together with the cc-pVTZ basis sets. ${ }^{76}$ The MP2 calculations were carried out using the Gaussian package of programs. ${ }^{77}$

Several basis sets were used in the OVGF calculations. ${ }^{7,16}$ Besides the cc-pVDZ basis, allowing for a comparison of the OVGF and ADC(3) results, the hierarchy of cc-pVnZ $(n=$ $\mathrm{D}, \mathrm{T}, \mathrm{Q}, 5)$ and aug-cc-pVnZ $(n=\mathrm{D}, \mathrm{T}, \mathrm{Q})$ correlation consistent basis sets ${ }^{76}$ with spherical representation of $d$-functions were also used.

The frozen-core approximation was adopted in the ADC(3) and OVGF calculations, that is, the carbon and nitrogen K-shell orbitals were not correlated. This frozen-core model has been adopted in the notation of the molecular orbitals throughout this paper.

The results of the OVGF calculations allow the convergence of the ionization energies with respect to systematic improvements in the basis set to be studied and to extrapolate to the CBS limit. Here we use the three-parameter exponential formula: ${ }^{78-80}$

$$
E(n)=E_{\mathrm{CBS}}+B e^{-\gamma n},
$$


where $n$ is the cardinal number of the basis set $(2,3,4,5$ for D, T, Q, and 5 sets, respectively), $E_{\mathrm{CBS}}$ is the estimated ionization energy for the CBS limit (as $\left.n \rightarrow \infty\right)$, and $B, \gamma$ are further parameters found by fitting Eq. (20) to the computed energies $E(n)$. This type of extrapolation is known to provide sufficiently reliable results, ${ }^{78-80}$ although some other techniques can be more advantageous for extrapolations of the ionization energies. ${ }^{69}$

The outer-valence vertical ionization energies were also computed using the EOM-IPCCSD method, ${ }^{19-22}$ as implemented in the Q-Chem program package. ${ }^{81}$ The aug-cc-pVTZ basis set ${ }^{76}$ was used in these calculations.

The restricted Hartree-Fock (RHF) and unrestricted Hartree-Fock (UHF) calculations were performed to obtain $\mathrm{HF}, \mathrm{RHF} / \triangle \mathrm{SCF}$, and $\mathrm{UHF} / \triangle \mathrm{SCF}$ ionization energies. The calculations were carried out using the Gaussian, ${ }^{77}$ Q-Chem, ${ }^{81}$ and Gamess ${ }^{75}$ program packages. In the $\triangle \mathrm{SCF}$ calculations, in addition to the ${ }^{1} \mathrm{~A}_{1}$ ground-state of neutral pyridine, the ${ }^{2} \mathrm{~A}_{1}\left(7 \mathrm{a}_{1}{ }^{-1}\right),{ }^{2} \mathrm{~A}_{2}\left(1 \mathrm{a}_{2}{ }^{-1}\right),{ }^{2} \mathrm{~B}_{1}\left(2 \mathrm{~b}_{1}{ }^{-1}\right),{ }^{2} \mathrm{~B}_{2}\left(5 \mathrm{~b}_{2}{ }^{-1}\right)$ states of the pyridine cation-radical were also considered.

In the " $\Delta "$ "-approach, the total energies of the initial and final states are computed, and the transition energies are then given as total energy differences. In the present work the $\Delta$-approach to ionization energies was applied at the $\triangle \mathrm{MP} 2$ (second-order Moller-Plesset $\mathrm{PT}), \triangle \mathrm{MP} 3$, and $\triangle \mathrm{MP} 4$ levels, as well as by using coupled cluster methods at the $\triangle \mathrm{CCD}$ (coupled cluster doubles), $\triangle \mathrm{CCSD}$, and $\triangle \mathrm{CCSD}(\mathrm{T})$ (singles and doubles with perturbative treatment of triples) levels. The calculations were carried out using the Gaussian ${ }^{77}$ program within the UHF and frozen-core frameworks.

The EOM-CC calculations of vertical ionization energies were carried out using the following models: CCSD, CC3 (CCSD model with approximate treatment of triple excitations), CCSDT (the full coupled cluster singles, doubles and triples model). Whereas the CCSD and CCSDT calculations were done within the EOM-CC approach for ionization energies (EOM-IP-CC) ${ }^{19-22}$ for the CC3 calculations the EOM-CC approach for the excitation energies (EOM-EE-CC3) was employed in which the continuum orbital approximation ${ }^{82}$ allows for the evaluation of ionization energies. The EOM-CC calculations were performed with the CFOUR program ${ }^{83}$ within the frozen-core framework. 
In order to obtain the adiabatic and 0-0 transition energies, the geometry optimization for the ${ }^{2} \mathrm{~A}_{1}\left(7 \mathrm{a}_{1}{ }^{-1}\right),{ }^{2} \mathrm{~A}_{2}\left(1 \mathrm{a}_{2}{ }^{-1}\right),{ }^{2} \mathrm{~B}_{1}\left(2 \mathrm{~b}_{1}{ }^{-1}\right)$, and ${ }^{2} \mathrm{~B}_{2}\left(5 \mathrm{~b}_{2}{ }^{-1}\right)$ cationic states was performed at the MP2 and CCSD levels of theory using the cc-pVTZ basis set. The geometry optimization for the ${ }^{2} \mathrm{~B}_{2}\left(5 \mathrm{~b}_{2}{ }^{-1}\right)$ state carried out using the UHF-based MP2 method failed. Regardless of the initial guess, large gradients were obtained which caused a reduction of the molecular symmetry to the $C_{1}$ point group. All cationic states then fall into the same irreducible representation, and the variational UHF method converges to the lowest state ${ }^{2} \mathrm{~A}_{2}\left(1 \mathrm{a}_{2}{ }^{-1}\right)$. The optimized geometric parameters for the ${ }^{2} \mathrm{~A}_{2}\left(1 \mathrm{a}_{2}{ }^{-1}\right)$ state were therefore obtained. The equilibrium electronic ground-state geometry of neutral pyridine was also computed using the same methods for a reference. For all states where MP2-optimized geometric parameters could be obtained, the harmonic vibrational frequencies were evaluated at the MP2 level of theory. The calculations were carried out using the Gaussian ${ }^{77}$ program within the UHF and frozen-core frameworks.

The second-order perturbation theory for the relaxation energy, presented in Sec. IID, was implemented as a supplement to our ADC software. ${ }^{74}$ The calculations were performed using the compact $6-31 \mathrm{G}^{*}$ basis set ${ }^{84}$ to simplify the analysis of the results.

The Gaussian Cube utility ${ }^{77}$ was used to generate the total electron densities $\rho(\mathbf{r})$ and the electron density differences $\Delta \rho(\mathbf{r})$. The $\Delta \rho_{n}^{N-1 / N}$ (r) function (Eq. (10)) was evaluated at the CCD level of theory within the UHF and frozen-core frameworks. The $\Delta \rho_{n}^{N-1(\mathrm{rel})}$ function (Eq. (11)) was generated from the RHF/ $\mathrm{SCF}$ and HF densities. The latter were obtained as follows: the neutral ground-state HF orbitals were given as the initial guess for the cationic-state RHF calculations and the SCF procedure was terminated before the onset of the iterations. The $\Delta \rho_{n}^{N-1 \text { (cor) }}$ function (Eq. (12)) was obtained using the CCD and UHF/ $\Delta \mathrm{SCF}$ densities. The cc-pVTZ basis set was used in these calculations. The GaussView program ${ }^{85}$ was employed to produce contour plots. The contour values suggested by GaussView, 0.001 , $0.002,0.004,0.008,0.02,0.04,0.08,0.2,0.4,0.8,2$, were adopted. The Molden software ${ }^{86}$ was used to plot the Dyson orbitals computed by our one-particle Green's function program ${ }^{74}$ at the $\mathrm{ADC}(3) / \mathrm{cc}-\mathrm{pVDZ}$ level of theory. 


\section{F. CMS-Xa calculations of photoionization cross-sections and photoelectron angular distributions}

Photoionization dynamics calculations were performed using the CMS-X $\alpha$ approach, following procedures similar to those described previously. ${ }^{87,88}$ Briefly, a model potential is constructed using overlapping, spherical atomic volumes, placed at centres determined from the MP2/cc-pVTZ optimized geometry, with atomic radii selected and scaled $(\times 0.85)$ as previously described. ${ }^{88}$ We then iterate to achieve a self-consistent ground state potential in which the exchange contribution to an effective one-electron potential is represented using the $\mathrm{X} \alpha$ local density approximation. A final state cation potential having the correct asymptotic Coulombic form was subsequently generated from this ground state neutral potential. Electron wavefunctions, expanded in a symmetry adapted basis of spherical harmonic functions, and with radial terms obtained by direct numerical integration, are then obtained in these potentials and used to construct the electric dipole photoionization matrix elements in an independent electron, frozen core approximation. Finally, photoionization cross-sections and photoelectron anisotropy parameters were computed from these matrix elements, and thus represent ionization of a fixed geometry, non-rotating, non-vibrating molecule.

The spherical harmonic basis expansions for the initial neutral state were truncated at values, $l_{\max }$, of 5,2 and 1 in, respectively, the outer molecule region, the non-hydrogenic atomic regions, and the hydrogen atoms regions. For the final continuum state, the outgoing electron can be scattered into higher $l$-waves by the anisotropic ion potential, and so the corresponding truncation limits were extended to $l_{\max }=9,4$, and 3 .

\section{EXPERIMENTAL APPARATUS AND PROCEDURE}

Photoelectron spectra of pyridine were recorded using a rotatable hemispherical electron energy analyzer and synchrotron radiation emitted from the Daresbury Laboratory storage ring. ${ }^{89}$ Detailed descriptions of the monochromator ${ }^{90}$ and the experimental procedure have been reported. ${ }^{91}$ 
The photoionization differential cross-section in the electric dipole approximation assuming randomly oriented targets and electron analysis in a plane perpendicular to the photon propagation direction, can be expressed in the form

$$
\frac{\mathrm{d} \sigma}{\mathrm{d} \Omega}=\frac{\sigma_{\text {total }}}{4 \pi}\left[1+\frac{\beta}{4}(3 \mathrm{P} \cos 2 \theta+1)\right]
$$

where $\sigma_{\text {total }}$ is the angle-integrated cross-section, $\beta$ is the photoelectron anisotropy parameter, $\theta$ is the photoelectron ejection angle relative to the major polarization axis and $\mathrm{P}$ is the degree of linear polarization of the incident radiation. At each photon energy, photoelectron spectra were recorded at $\theta=0^{\circ}$ and $\theta=90^{\circ}$, thus allowing the anisotropy parameter to be determined once the polarization had been deduced. The degree of polarization was determined by recording $\mathrm{Ar} 3 \mathrm{p}$ and $\mathrm{He} 1 \mathrm{~s}$ photoelectron spectra as a function of photon energy, and using the well-established $\beta$-parameters for these gases. ${ }^{91,92}$

The spectra were analyzed by dividing the binding energy range into the regions specified in Table I to obtain photoelectron anisotropy parameters and $\beta$-independent branching ratios. The branching ratio for a specific region is defined as the intensity in that particular region divided by the sum of the intensity in all the energetically accessible regions. Since the binding energies for some of the orbitals are very similar, the corresponding photoelectron bands overlap to some extent, and for these bands a complete separation between the ionic states is not possible.

The branching ratios (not shown) for the outer valence shell were used to derive absolute photoionization partial cross-sections (regions $1-8$, Table I) as follows. First, the absolute total photoionization cross-section was obtained as the product of the absolute photoabsorption cross-section ${ }^{93}$ and the photoionization quantum efficiency. ${ }^{93}$ Secondly, the absolute photoionization partial cross-section for a particular region (Table I) was then determined by multiplying the appropriate branching ratio with the absolute total photoionization cross-section. 


\section{RESULTS AND DISCUSSION \\ A. Pyridine molecular orbitals}

According to our HF calculations, the ground-state valence-shell electron configuration of pyridine can be written using $\mathrm{C}_{2 \mathrm{v}}$ point group notation as

Inner valence: $1 \mathrm{a}_{1}^{2} 2 \mathrm{a}_{1}^{2} 1 \mathrm{~b}_{2}^{2} 3 \mathrm{a}_{1}^{2} 2 \mathrm{~b}_{2}^{2}$

Outer valence: $4 a_{1}^{2} 3 b_{2}^{2} 5 a_{1}^{2} 4 b_{2}^{2} 6 a_{1}^{2} 1 b_{1}^{2} 5 b_{2}^{2} 7 a_{1}^{2} 2 b_{1}^{2} 1 a_{2}^{2}$

where the core orbitals are excluded from the orbital numbering scheme. The character of the outer-valence orbitals can be understood from the Mulliken atomic populations given in Table II.

The MOs can be assigned as $\sigma$ - and $\pi$-type, as is usual for aromatic molecules. The aromatic system comprises three $\pi$-orbitals $\left(1 b_{1}, 2 b_{1}, 1 a_{2}\right)$. A characteristic feature of the pyridine molecule is the non-bonding $\sigma$-type lone-pair orbital of the nitrogen atom $\left(7 \mathrm{a}_{1}\right)$. This orbital appears in the spectrum at a low binding energy, although the exact location remains uncertain. According to the Mulliken populations (Table II), the n $\sigma_{N L P}$ orbital shows a substantial localization on nitrogen, although some of the electron density is delocalized over the hydrogen atoms. The other orbitals forming the outer- and inner-valence shells of pyridine are essentially $\sigma$-type bonding orbitals with significant delocalization over the ring and hydrogen atoms.

\section{B. Vertical ionization energies}

The energies and intensities of the vertical transitions belonging to the outer-valence region of the ionization spectrum, computed using various methods, are listed in Table III. The theoretical results are obtained using the HF, OVGF, ADC(3) and EOM-IP-CCSD methods in combination with the cc-pVDZ and aug-cc-pVTZ basis sets. Experimental values are also given for comparison.

The basis set effect can be studied in more detail using the data given in Table IV, comparing the OVGF results for the aug-cc-pVnZ and cc-pVmZ series up to $n=\mathrm{Q}$ and $m=5$. Since the accuracy of the OVGF results above $16 \mathrm{eV}$ is not sufficient for a reliable extrapolation, due to increased configuration interaction effects in this energy region, the data 
for the $5 a_{1}, 3 b_{2}$ and $4 a_{1}$ orbitals were not included. Beyond the cc-pVTZ level, the results are nearly converged, so that the cc-pVTZ and aug-cc-pVTZ sets can be used for a stringent comparison with both experiment and between different theoretical schemes. The cc-pVDZ basis set, on the other hand, is more appropriate for a qualitative description of the spectrum yielding ionization energies typically $0.25 \mathrm{eV}$ below the basis set limit.

Considerable corrections to the HF ionization energies can be seen in the results of the correlated methods for almost all states. Exceptions are the ionization energies of the outermost $\pi$-orbitals, $2 b_{1}$ and $1 a_{2}$, which are surprisingly stable with respect to the theoretical treatment used. There are two groups of orbitals, $\left(1 a_{2}, 2 b_{1}, 7 a_{1}\right)$ and $\left(5 a_{1}, 3 b_{2}\right)$, where the energetic ordering changes with respect to the HF predictions when more accurate methods are employed. Limitations of the one-electron approximation also play a role in the $1 b_{1}^{-1}$, $3 b_{2}{ }^{-1}$, and $4 a_{1}^{-1}$ states, where reduced intensities of the corresponding transitions are predicted at the $\mathrm{ADC}(3)$ level. Here, configuration interaction in the final cationic states becomes important, and the HF and OVGF methods are no longer strictly appropriate. This, for example, can be seen in the large discrepancy between the OVGF and ADC(3) results for the $3 \mathrm{~b}_{2}{ }^{-1}$ state, which otherwise are in good mutual agreement. Typically here the presence of satellites, acquiring their intensity from the respective $1 \mathrm{~h}$ main state, can be expected. ${ }^{4}$ Overall, the one-particle picture of ionization ${ }^{4}$ holds to a good approximation over the entire outer-valence region.

In general, the present $\mathrm{ADC}(3), \mathrm{OVGF}$, and EOM-IP-CCSD results are fairly consistent with the experimental data. Disparities, such as those for ionization of the $3 b_{2}$ and $4 a_{1}$ orbitals, can most likely be explained by the possibility that the measured peak positions do not strictly match the vertical ionization energies. The two quantities can deviate from each other due to extended yibrational progressions and overlapping spectral structures. As already mentioned, the first band in the photoelectron spectrum encompasses two closely spaced transitions due to the $1 \mathrm{a}_{2}(\pi)$ and $7 \mathrm{a}_{1}\left(\sigma_{\mathrm{N}} \mathrm{LP}\right)$ orbitals. In view of the error margins in the computational results (Table III), the correct energetic ordering of these states becomes an issue. Whereas the ADC(3) and OVGF methods predict the state ordering to be $1 a_{2}(\pi)^{-1}$ followed by $7 a_{1}\left(\sigma_{N} L P\right)^{-1}$ at slightly higher energy, the EOM-IP-CCSD calculations indicate the reverse order. 


\section{Cationic states ${ }^{2} A_{1}\left(7 a_{1}{ }^{-1}\right),{ }^{2} A_{2}\left(1 a_{2}{ }^{-1}\right),{ }^{2} B_{1}\left(2 b_{1}{ }^{-1}\right)$, and ${ }^{2} B_{2}\left(5 b_{2}{ }^{-1}\right)$}

The lack of agreement amongst the theoretical methods with respect to the low-energy part of the ionization spectrum calls for further attention. To address this issue, in Table V we list the results for the four lowest vertical ionization energies obtained in the present work using different computational approaches, as well as those from previous studies. ${ }^{35,40,42,46}$ As can be seen, the difficulty arises mainly from the large variation in the estimates for the nitrogen LP orbital, $7 \mathrm{a}_{1}\left(\sigma_{\mathrm{N}} \mathrm{LP}\right)$, whose ionization energy depends strongly on the method used.

The $7 \mathrm{a}_{1}\left(\sigma_{\mathrm{N} L P}\right)$ ionization energy, being grossly overestimated at the HF level $(11.37 \mathrm{eV})$, is shifted down by $2.73 \mathrm{eV}$ to $8.64 \mathrm{eV}$ at the RHF/ $\mathrm{SCF}$ level of theory. The corresponding shifts for the other orbitals, $1 a_{2}(\pi), 2 b_{1}(\pi), 5 b_{2}(\sigma)$, are less pronounced $(0.85$, $0.96,0.86 \mathrm{eV}$, respectively). As a result, the $7 \mathrm{a}_{1}\left(\sigma_{\mathrm{N} L P}\right)^{-1}$ state moves from the third lowest place in the spectrum to the second, and becomes nearly degenerate with the lowest state, $1 \mathrm{a}_{2}(\pi)^{-1}$. The UHF/ $\mathrm{SCF}$ method provides qualitatively similar results, but yields even smaller ionization energies than those determined in the RHF treatment, due to the lower energies of the cationic-states obtained at the UHF level.

Methods going beyond the HF level treat both electron correlation and orbital relaxation, and shift the $7 \mathrm{a}_{1}\left(\sigma_{\mathrm{N}} \mathrm{LP}\right)^{-1}$ state to a lower energy with respect to the HF value. As a result, the $7 \mathrm{a}_{1}\left(\sigma_{\mathrm{N} / \mathrm{P}}\right)^{-1}$ state becomes the second lowest state in the $\triangle \mathrm{MP} 2, \triangle \mathrm{MP} 3, \triangle \mathrm{MP} 4$, $\triangle \mathrm{CCD}, \triangle \mathrm{CCSD}, \mathrm{OVGF}$, and $\mathrm{ADC}(3)$ treatments, and the lowest in the results of the other correlated methods in Table V. The energy gap between the $7 \mathrm{a}_{1}\left(\sigma_{\mathrm{NLP}}\right)^{-1}$ and $1 \mathrm{a}_{2}(\pi)^{-1}$ states is significantly reduced, especially at the $\triangle \mathrm{CCSD}, \triangle \mathrm{CCSD}(\mathrm{T}), \mathrm{EOM}-\mathrm{IP}-\mathrm{CCSD}$, and CASPT2 levels of theory/where the two states are nearly degenerate.

At the highest level of theory (EOM-IP-CCSDT) employed in the present work, the $7 a_{1}\left(\sigma_{N} L P\right)^{-1}$ state is predicted to lie vertically below the $1 a_{2}(\pi)^{-1}$ state by $0.12 \mathrm{eV}$. The EOMIP-CCSDT/cc-pVTZ results, owing to the high-level of the CCSDT approximation and the cc-pVTZ basis (Table IV), provide a very accurate description of the electronic structure.

Using the EOM-IP-CCSDT/cc-pVTZ results as benchmarks for the other computational schemes in Table $\mathrm{V}$, one notices that the $\pi$-states, in general, converge faster with the level of 
theoretical treatment than do the $\sigma$-states. According to the absolute mean error and the maximal errors, the EOM-IP-CC3 and EOM-IP-CCSD results are in best agreement with the EOM-IP-CCSDT benchmarks. The CC3 model is clearly superior to the CCSD method with respect to the "difficult" $7 a_{1}\left(\sigma_{N L P}\right)^{-1}$ and $5 b_{2}(\sigma)^{-1}$ states, while for the $\pi$-type states the two schemes show roughly equivalent performance. The $\Delta \operatorname{CCSD}(\mathrm{T})$ scheme shows nearly the same numerical performance as that for the EOM-IP-CCSD scheme. Slightly larger mean absolute errors of $0.1-0.2 \mathrm{eV}$ occur for the ADC(3), OVGF, and $\triangle \mathrm{CCSD}$ schemes. Interestingly, the OVGF results appear to be somewhat more accurate than the ADC(3) results. As expected, the simple $\triangle \mathrm{CCD}$ model is the least satisfactory among the $\mathrm{CC}$ and GF methods. It is noticeable that the $\triangle \mathrm{CCD}$ ionization energies are almost identical to those obtained using the $\triangle \mathrm{MP} 3$ scheme. The accuracy afforded by the $\Delta \mathrm{MP} n$ schemes is relatively poor in comparison with the CC and GF methods. A better level of accuracy is attained in the third-order GF schemes, ADC(3) and OVGF, and also in the CCSD scheme which, however, is consistent only through second order.

Despite the differences in the basis sets and geometrical parameters, the results from previous theoretical studies are quite consistent with the present calculations. The ADC(3) vertical ionization energies obtained by Moghaddam et al. ${ }^{35}$ are systematically $0.2-0.3 \mathrm{eV}$ lower than the present $\operatorname{ADC}(3)$ values (Table V). For the $\pi$-type states, $1 a_{2}{ }^{-1}$ and $2 b_{1}{ }^{-1}$, the $\mathrm{ADC}(3)$ results $^{35}$ are very similar to the SAC-CI data of Wan et al. ${ }^{42}$ However, for the $\sigma$-type states the vertical ionization energies obtained in the latter work are somewhat too small. Similarly, the MRD-CI results of Walker et al. ${ }^{40}$ clearly underestimate the ionization energy of the $7 \mathrm{a}_{1}\left(\sigma_{\mathrm{N}} \mathrm{LP}\right)$ orbital, predicting it to be only $8.84 \mathrm{eV}$; the overall accuracy is similar to that of the SAC-CI calculations. The CASPT2 results of Lorentzon et al. ${ }^{46}$ (unavailable for $5 b_{2}(\sigma)^{-1}$ state) appear to be very accurate and show only minor deviations from the EOM-IPCCSDT energies.

In view of the apparent complexity of the lowest cationic states of pyridine, a more detailed analysis seems appropriate. First, one can look at the Dyson orbitals, which represent many-electron effects in orbital form, visualizing the molecular domain left by the emitted electron (Figure 2). The Dyson orbitals for the $1 a_{2}(\pi)^{-1}$ and $2 b_{1}(\pi)^{-1}$ states closely resemble 
Publishing the corresponding canonical HF molecular orbitals, both being of typical $\pi$-character. The Dyson orbital for the $7 \mathrm{a}_{1}\left(\sigma_{\mathrm{N} L P}\right)^{-1}$ state is also rather similar to the corresponding HF MO. Its lone-pair character is clearly reflected in the characteristic density maximum, directed perpendicular to the ring plane, at the nitrogen site. For the $5 b_{2}(\sigma)^{-1}$ state the Dyson and HF orbitals describe $\sigma$-bonding of the $\mathrm{N}-\mathrm{C}_{2}, \mathrm{C}_{3}-\mathrm{C}_{4}$, and $\mathrm{C}-\mathrm{H}$ atoms.

More information can be gained from the density difference plots comparing the total correlated electron density in the electronic ground state of neutral pyridine and its cationic states. Since total densities cannot be obtained in the ADC approach, they were evaluated using the CCD method for both the initial and final states (Figure 2). This should be sufficient for a qualitative analysis. The regions in the density difference plots shown in blue are depleted as a result of electron emission. The shape of these areas agrees with the shape of the Dyson orbitals when the phase structure is disregarded in the latter.

The Dyson orbitals and the density difference plots are equally useful for visualizing regions of decreased electron density. The density difference plots, however, also give an idea of the density redistribution in the cations due to orbital relaxation and electron correlation. In Figure 2, the regions plotted in red are characterized by increased electron population and reflect the rearrangements taking place in the cationic states. As can be seen, the areas of increased density are compactly located in the central part of the molecule, mainly on the bonds forming the six-membered ring. This can be understood in that the remaining density tends to strengthen the bonds in the cation-radicals compensating for the loss of an electron.

The observed rearrangements can also be interpreted as a screening of the positive charge of the hole generated by the removal of an electron. For the $\pi$-type final states, ${ }^{2} \mathrm{~A}_{2}$ $\left(1 a_{2}^{-1}\right)$ and ${ }^{2} B_{1}\left(2 b_{1}^{-1}\right)$, the screening effect is observed in the molecular plane, which is the nodal plane of the $\pi$-orbitals. For states of $\sigma$-symmetry, ${ }^{2} \mathrm{~A}_{1}\left(7 \mathrm{a}_{1}{ }^{-1}\right)$ and ${ }^{2} \mathrm{~B}_{2}\left(5 \mathrm{~b}_{2}{ }^{-1}\right)$, in contrast, some increase of electron density can also be seen above (and below) the molecular plane. 


\section{Orbital relaxation and electron correlation effects}

It has yet to be explained why the ground-state HF orbital (Koopmans' theorem) provides such a poor description of the $7 \mathrm{a}_{1}\left(\sigma_{\mathrm{N}} \mathrm{LP}\right)^{-1}$ state, necessitating a large correction at the higher computational levels. It is instructive to note that the $\triangle \mathrm{SCF}$ treatment yields quite reasonable estimates for the relative ionization energies (or spacing) associated with the three lowest states, $1 \mathrm{a}_{2}(\pi)^{-1}, 7 \mathrm{a}_{1}\left(\sigma_{\mathrm{N} L P}\right)^{-1}$ and $2 \mathrm{~b}_{1}(\pi)^{-1}$. The $\Delta$ SCF scheme fully accounts for orbital relaxation but disregards electron correlation, which indicates that relaxation is a crucial factor in the description of the $7 \mathrm{a}_{1}\left(\sigma_{\mathrm{N}} \mathrm{LP}\right)^{-1}$ state. Electron correlation, on the other hand, improves the absolute ionization energies by shifting both the neutral ground state and the cationic states to lower energy. The resulting correction, defined by these two shifts, is state specific.

\section{Orbital relaxation}

In order to see how relaxation changes the localization properties of the $7 \mathrm{a}_{1}\left(\sigma_{\mathrm{N} \text { LP }}\right)$ and other molecular orbitals, in Table VI we compare their Mulliken atomic populations in the electronic ground state (closed shells) and in the respective cation radicals (half-occupied shells). Table VI shows that the Mulliken atomic population of the $7 \mathrm{a}_{1}\left(\sigma_{\mathrm{N}} \mathrm{LP}\right)$ MO changes dramatically upon ionization. The nitrogen character increases by about $25 \%$, from $62 \%$ in neutral pyridine to $88 \%$ in the pyridine cation-radical. The $7 \mathrm{a}_{1}\left(\sigma_{\mathrm{N}} \mathrm{LP}\right) \mathrm{MO}$ is significantly more localized on nitrogen in the cationic state than in the (neutral) ground state. This can be seen not only in the decreased contribution of the carbon atoms, but also in a quite substantial (7\%) decrease in the contributions of the hydrogen atoms. By contrast, the $\pi$-orbitals, $1 \mathrm{a}_{2}(\pi)$ and $2 \mathrm{~b}_{1}(\pi)$, hardly change upon ionization, and largely retain their localization properties in the cation-radical. The localization properties of the $5 b_{2}(\sigma)$ orbital change moderately. Here a certain decrease of $\mathrm{N}$ - and $\mathrm{C}_{2^{-}}$character and the corresponding increase of $\mathrm{C}_{3^{-}}$and $\mathrm{C}_{4^{-}}$ character occurs.

The changes in the total electronic density distribution caused by relaxation can be visualized by the density difference plots (Figure 3 ) for the $7 a_{1}\left(\sigma_{N} L P\right)$ and $5 b_{2}(\sigma)$ orbitals. Similar plots for the $1 a_{2}(\pi)$ and $2 b_{1}(\pi)$ orbitals, together with above-plane density difference 
plots for all four orbitals, are shown in Figure S1 in Supplementary Material. These plots compare the cationic states in terms of the unrelaxed (neutral ground-state HF) density and the fully relaxed density obtained from the ROHF calculations for the state under consideration.

For the ${ }^{2} \mathrm{~A}_{1}\left(7 \mathrm{a}_{1}^{-1}\right)$ state the density difference surface is very steep, especially in the vicinity of the nitrogen atom. This implies that a strong rearrangement of the electron density due to relaxation takes place. When relaxation is taken into account, more electron density is withdrawn from the area surrounding the nitrogen atom, whereas there is an increase in the electron density on the molecular peripheral domains including the hydrogen atoms and $\mathrm{C}-\mathrm{H}$ bonds. The in-plane and above-plane cross-sections show a similar relaxation pattern, although - as expected for states of $\sigma$-symmetry - the effect is much weaker above the molecular plane. This description is in agreement with the picture based on the Mulliken atomic populations given in Table VI, predicting a more pronounced lone-pair character for the $7 \mathrm{a}_{1}$ orbital at the level of the $\triangle \mathrm{SCF}$ treatment.

The density difference surfaces of the $\pi$-states, ${ }^{2} \mathrm{~A}_{2}$ and ${ }^{2} \mathrm{~B}_{1}$, are rather flat (Figure $\mathrm{S} 1$ ), which means that there is no pronounced relaxation effect. For the $\pi$-states, the density difference above the molecular plane has to be considered. It shows density redistribution patterns similar to the shapes of the corresponding $1 a_{2}$ and $2 b_{1}$ orbitals. Interestingly, there is also a notable in-plane relaxation effect leading to quite different and substantially more symmetric density redistribution patterns. In both cases, the in-plane electron density is transferred from the six-membered ring towards the hydrogen atoms.

Strong in-plane relaxation effects can be seen for the ${ }^{2} \mathrm{~B}_{2}\left(5 b_{2}{ }^{-1}\right)$ state (Figure 3). Here the electron density is transferred from the bonds adjacent to the $\mathrm{C}_{4}$ atom to the bonds on the opposite side of the molecule adjacent to nitrogen. The in- and above-plane reorganization effects differ somewhat, as can be seen from the respective surfaces (Figure S1).

\section{Electron correlation}

The orbital relaxation effects in the pyridine cation-radicals considered above are characterized by a strong non-local density redistribution, resembling to a certain extent intra- 
molecular charge transfer. In contrast, when electron correlation is taken into account the resulting changes to the electron distribution are essentially local. This is evident from the characteristic pattern in the total electron density difference maps (Figures 3 and S2 in Supplementary Material) obtained by comparing the relaxed UHF densities for the cationic states with the densities derived from CCD computations using the UHF orbitals. For all four states considered here, one sees that electron correlation introduces local corrections which decrease the density on the chemical bonds and increase the density near atoms. This leads to a more balanced electron density distribution, compared to the case when only orbital relaxation effects are taken into account, and is in agreement with the earlier findings concerning the role of the electron correlation effects.

Considerable in-plane density redistribution due to electron correlation can be seen in the $\pi$-states, ${ }^{2} \mathrm{~A}_{2}$ and ${ }^{2} \mathrm{~B}_{1}$ (Figure $\mathrm{S} 2$ ). As already mentioned, the correlation induced changes modify the local bonding situation. The above-plane cuts of the density difference maps do not facilitate a simple interpretation, but clearly indicate that for both $\pi$ - and $\sigma$-type states correlation related changes in the electron density arise throughout the entire molecular environment, including regions distant from the ring plane.

The electron correlation effects for the ionization energies, as obtained by the $\triangle \mathrm{CCD}$ approach, are quite considerable, amounting to $1.57,1.18,1.30,0.81 \mathrm{eV}$ for the $7 \mathrm{a}_{1}\left(\sigma_{\mathrm{LP}}\right)^{-1}$, $1 a_{2}(\pi)^{-1}, 2 b_{1}(\pi)^{-1}, 5 b_{2}(\sigma)^{-1}$ states, respectively (Figures 3 and S2). These corrections are roughly of the same order of magnitude as the relaxation shifts $(-2.73,-0.85,-0.96$, $-0.86 \mathrm{eV}$, respectively), but have the opposite sign (Figures 3 and $\mathrm{S} 1$ ). Except for the $7 a_{1}\left(\sigma_{L P}\right)^{-1}$ state, this leads to a rather effective compensation of the two effects, resulting in modest overall corrections (Figure S3 in Supplementary Material). For the ${ }^{2} \mathrm{~A}_{1}\left(7 \mathrm{a}_{1}{ }^{-1}\right)$ state, however, the relaxation effect is much more substantial than the correlation contribution, so the total correction is large $(-1.16 \mathrm{eV})$. The electron density difference maps (Figure $\mathrm{S} 3$ in Supplementary Material), incorporating both effects, support these findings. One can therefore state that the neutral ground-state HF description of the $\sigma_{\mathrm{LP}} \mathrm{MO}$ in pyridine - and most likely in other heteroaromatic compounds - is inappropriate for the associated cationic states. 


\section{Perturbation treatments}

As addressed in Sec. IID, the $\triangle \mathrm{SCF}$ calculations can be analyzed by perturbation theory. The results of a PT2 analysis for the four highest occupied orbitals are given in Table VII. The $\triangle \mathrm{SCF}$ and HF ionization energies are also listed, establishing the full relaxation energy, $\Delta E^{R}(\Delta \mathrm{SCF})$, according to Eq. (13). As can be seen, the PT2 approach recovers the relaxation energy surprisingly well. The mean error for the $\pi$ - and $\sigma$-type states is only 0.2 and $0.1 \mathrm{eV}$, respectively, with $\Delta E^{R}$ being overestimated in the former and underestimated in the latter. The IPs derived from PT2 and $\triangle \mathrm{SCF}$ are in good agreement as well.

The five largest terms in the PT2 expansions of $\Delta E_{k}^{R}$ (Eq. (16)) for the $7 \mathrm{a}_{1}\left(\sigma_{\mathrm{N} L P}\right)$, $1 a_{2}(\pi), 2 b_{1}(\pi)$, and $5 b_{2}(\sigma)$ orbitals are shown in Table VIII, together with the dominant $S_{1}$ terms (Eq. (18)), the $(k k \mid a i)$ integrals, and the $\left(\varepsilon_{a}-\varepsilon_{i}\right)$ orbital energy differences (the latter two quantities appear in the nominator and denominator, respectively, of $S_{1}$ ). Inspection of the terms listed in Table VIII shows that there are obvious differences between the cationic states of $\sigma_{N L^{-}}, \pi$-, and $\sigma$-type. According to our results, the $\sigma_{\mathrm{N} \text { LP }}$ relaxation is driven mainly by the two-electron integrals of $\left(\sigma_{N L P} \sigma_{N L P} \mid \pi \pi^{*}\right) \equiv\left\langle\pi\left|\hat{J}_{\sigma N L P}\right| \pi^{*}\right\rangle$ type describing the coupling of the $\sigma_{\mathrm{N} L P}$ charge to the electron density distributed in the $\pi$ and $\pi^{*}$ orbitals. The large relaxation effect in the $7 \mathrm{a}_{1}\left(\sigma_{\mathrm{LP}}\right)^{-1}$ state is reflected in the substantial magnitude of that Coulomb integral. Also, the small $\pi-\pi^{*}$ energy gap plays an important role (Table VIII). This finding implies that the $\pi$-system plays an active role in the screening of the $\sigma_{\mathrm{N} \text { LP-hole }}$ and in the relaxation accompanying the ionization of an $\sigma_{\mathrm{N} L P}$ electron (Eq. (14)). This is also in agreement with the density/difference plots demonstrating a considerable above-plane relaxation component for ionization of the $\sigma_{\mathrm{LP}}$-orbital. Indeed, according to our results, more than $50 \%$ of the relaxation energy of the $\sigma_{\mathrm{LP}}$ orbital is due to the $\pi$-system contribution (as indicated by the $\Delta E_{\pi}^{R}$ value in Table VII). In contrast, the $\pi$-system contributes only $28 \%$ to the relaxation energy of the $5 \mathrm{~b}_{2}(\sigma)^{-1}$ state (Table VII), which clearly shows here a distinctly different relaxation mechanism. For the $\pi$-type states, $1 a_{2}(\pi)^{-1}$ and $2 b_{1}(\pi)^{-1}$, the main relaxation effect appears to be due to the $\sigma$-system, since the $\pi$-system supplies, in both cases, only $11 \%$ of the relaxation 
energy (Table VII). Thus, the most important two-electron integrals here are of $\left(\pi \pi \mid \sigma \sigma^{*}\right) \equiv\left\langle\sigma\left|\hat{J}_{\pi}\right| \sigma^{*}\right\rangle$ type (Table VIII).

An important distinction between the relaxation mechanism in the $7 \mathrm{a}_{1}\left(\sigma_{\mathrm{NLP}}\right)^{-1}$ state and that in the $1 \mathrm{a}_{2}(\pi)^{-1}, 2 \mathrm{~b}_{1}(\pi)^{-1}$, and $5 \mathrm{~b}_{2}(\sigma)^{-1}$ states is the existence of a single dominant term in the PT2 expansion (Table VIII). According to our results the largest $S$ contribution for the $7 \mathrm{a}_{1}\left(\sigma_{\mathrm{N} L P}\right)^{-1}$ state provides $24 \%$ of the total relaxation energy, but only 13,8 , and $10 \%$ in the $1 \mathrm{a}_{2}(\pi)^{-1}, 2 \mathrm{~b}_{1}(\pi)^{-1}$, and $5 \mathrm{~b}_{2}(\sigma)^{-1}$ states, respectively. In the latter cases no single major contribution can be identified; the individual contributions to the relaxation energy are relatively small as a consequence of small matrix elements in the nominators and large energy gaps in the denominators.

From the above considerations it is clear that the $\pi$-system, with the low-lying unoccupied $\pi^{*}$-MOs having similar localization properties as the occupied $\pi$-MOs, is essential for an efficient screening of the no-holes (the pairs of orbitals $2 b_{1}(\pi)-3 b_{1}\left(\pi^{*}\right)$ and $1 a_{2}(\pi)-2 a_{2}\left(\pi^{*}\right)$ fulfill this requirement). On the other hand, the no-orbital is well localized, so that large $\left\langle\pi\left|\hat{J}_{\mathrm{n \sigma}}\right| \pi^{*}\right\rangle$ integrals are obtained. Large-magnitude $\left\langle\pi\left|\hat{J}_{k}\right| \pi^{*}\right\rangle$ matrix elements, similar to those discussed here in relation to valence shell ionization, occur in K-shell ionization, where $k$ are highly localized 1s-MOs. Huge relaxation shifts are typical for such core-hole states. $^{66}$

The charge screening in the $\sigma_{\mathrm{LP}} \mathrm{MO}$ by the $\pi$-electrons is accomplished by $\pi$ - $\pi^{*}$ excitations. The relaxation effects are generally taken into account via electron interaction of $1 h$-states with $2 h-1 p-, 3 h-2 p$ - and higher excited states. Through first order of many-body perturbation theory, the interaction of the $1 h$-state $\left|\Phi_{k}\right\rangle$ and $2 h$-1p-state $\left|\Phi_{k j a}\right\rangle$ (representing a result of single electron excitation from occupied orbital $j$ to unoccupied orbital $a$ with respect to $\left.\left\langle\Phi_{k}\right\rangle\right)$ reads

$$
\left\langle\Phi_{k}\left|\hat{H}_{I}\right| \Phi_{k j a}\right\rangle=(k a \mid j k)-(k k \mid j a)
$$

where the usual Møller-Plesset partitioning $\hat{H}_{I}=\hat{H}-\hat{H}_{0}$ of the Hamiltonian $\hat{H}$ into the zero-order Hartree-Fock part $\hat{H}_{0}$ and the interaction $\hat{H}_{I}$ is assumed. The second term on the 
right-hand side of Eq. (22) is readily identified as the Coulomb integral $\left\langle j\left|\hat{J}_{k}\right| a\right\rangle$ discussed above in the context of the PT2 relaxation energy analysis.

The first-order interaction of the $1 h$ - and $2 h$-1p-states is explicitly taken into account in the $\mathrm{ADC}(3)$ and EOM-IP-CCSD schemes and contributes to the treatment of the orbital relaxation effects. Indeed, inspection of our ADC(3)/6-31G results for the $7 a_{1}\left(\sigma_{N} L_{P}\right)^{-1}$ state shows that the total weight of the $2 h-1 p$ configurations in the ADC(3) eigenvector amounts to $11 \%$, and the most important of these configurations are: $7 a_{1}^{-1} 2 b_{1}^{-1} 3 b_{1}, 7 a_{1}^{-1} 2 b_{1}^{-1} 4 b_{1}$, $7 a_{1}^{-1} 1 a_{2}^{-1} 2 a_{2}$ (that is, the excitations from the occupied $2 b_{1}$ and $1 a_{2} \pi$-orbitals into the virtual $3 b_{1}, 4 b_{1}$, and $2 a_{2} \pi^{*}$-orbitals). The extension of the basis set from $6-31 \mathrm{G}$ to cc-pVTZ hardly influences the results. In the higher-order EOM-IP-CC3 and EOM-IP-CCSDT schemes, the interaction of the $1 h$ - and $3 h-2 p$ - states comes into play. This improves the treatment of the orbital relaxation effects and, consequently, the results for the cationic states where such effects are important. Within the ADC hierarchy a similar improvement is expected at the level of the ADC(4) scheme, the proper implementation of which is still not available.

\section{E. Geometric structure and vibrational analysis}

Having discussed the vertical ionization energies of the four lowest cationic states, we now look at the geometric structure and vibrations. The optimized geometrical parameters for the ${ }^{2} \mathrm{~A}_{1}\left(7 \mathrm{a}_{1}{ }^{-1}\right),{ }^{2} \mathrm{~A}_{2}\left(1 \mathrm{a}_{2}^{-1}\right)$, and ${ }^{2} \mathrm{~B}_{1}\left(2 \mathrm{~b}_{1}^{-1}\right)$ states, obtained at the MP2 and CCSD levels using the cc-pVTZ basis set, are given in Tables IX and X, respectively. MP2 results for the ${ }^{2} \mathrm{~B}_{2}\left(5 \mathrm{~b}_{2}{ }^{-1}\right)$ state could not be obtained, since here the optimization procedure converged to the lower-lying ${ }^{2} \mathrm{~A}_{2}\left(1 \mathrm{a}_{2}{ }^{-1}\right)$ state, but CCSD/cc-pVTZ results for this state are included in Table X. The MP2 harmonic vibrational frequencies for the ${ }^{2} \mathrm{~A}_{1}\left(7 \mathrm{a}_{1}{ }^{-1}\right),{ }^{2} \mathrm{~A}_{2}\left(1 \mathrm{a}_{2}{ }^{-1}\right)$ and ${ }^{2} \mathrm{~B}_{1}\left(2 \mathrm{~b}_{1}{ }^{-1}\right)$ states are listed in Table XI.

The geometrical parameters predicted by the MP2 and CCSD methods are in fair agreement with each other. Whereas for neutral pyridine in its electronic ground state the MP2 method predicts slightly larger bond lengths than those obtained using the CCSD method, the opposite trend is observed for the cationic states. The largest inconsistencies are found for the ${ }^{2} \mathrm{~A}_{1}\left(7 \mathrm{a}_{1}{ }^{-1}\right)$ state, where differences up to $0.02-0.03 \AA$ occur for the C-C and C-N 
bond lengths. For both computational schemes, the bond lengths in the neutral ground-state are slightly smaller than the experimental values. ${ }^{96}$ Consistent results are obtained for the angles, and agree well with the experimental data. ${ }^{96}$ The neutral ground-state frequencies calculated in this work using the MP2 method agree very well with the experimental data of DiLella et al. ${ }^{97}$ The calculated normal modes are consistent with their classical definitions given by Langseth and Lord $^{98}$ and by Zerbi et al. ${ }^{99}$ The original notations of Langseth and Lord were therefore adopted, while some insight into the nature of the vibrations can be gained from the qualitative descriptions introduced by Kumar et al. ${ }^{100}$ also given in Table XI.

The vibrational frequencies of the cationic states are less well studied but Lee et al. ${ }^{39}$ have calculated B3LYP/cc-pVTZ frequencies, differing from those of the neutral ground state, for the ${ }^{2} \mathrm{~A}_{1}\left(7 \mathrm{a}_{1}^{-1}\right)$ state. In our calculation for the ${ }^{2} \mathrm{~B}_{1}\left(2 \mathrm{~b}_{1}^{-1}\right)$ state two imaginary frequencies (of $b_{1}$ and $b_{2}$ symmetry) were obtained. This means that here the symmetric $C_{2 v}$ molecular structure represents a saddle point on the potential energy surface, and one can expect that certain in-plane and out-of-plane distortions of the molecular configuration will lead to structures with lower total energy. Further studies are necessary to ascertain whether this result is an artifact of the method used, and whether the structures with reduced symmetry are bound.

\section{F. Assignment of the spectrum \\ 1. Vertical ionization}

The theoretical ionization spectrum of pyridine computed at the ADC(3)/cc-pVDZ level of theory is shown in Figure 4, together with a complete valence shell photoelectron spectrum recorded at a photon energy of $80 \mathrm{eV}$, and at $\theta=0^{\circ}$. The present theoretical spectrum agrees well with previous $\operatorname{ADC}(3)$ calculations. ${ }^{35,47}$ It reproduces the main features observed in the experimental spectrum and therefore can be used for qualitative assignments. The numerical data for the outer-valence region can be found in Tables III, IV and V.

According to the ADC(3) results, the photoelectron band occurring between 29.1 $10.3 \mathrm{eV}$ is formed by the closely spaced transitions into the $1 \mathrm{a}_{2}(\pi)^{-1}$ and $7 \mathrm{a}_{1}\left(\sigma_{\mathrm{N} \mathrm{LP}}\right)^{-1}$ states. This is in agreement with the results of the other theoretical methods used in the present work 
Publishing and in the previous studies (Table V). However, the vertical ordering of the $1 \mathrm{a}_{2}(\pi)^{-1}$ and $7 \mathrm{a}_{1}\left(\sigma_{\mathrm{N} L P}\right)^{-1}$ states cannot be determined unambiguously since the energy separation is smaller than the accuracy margin afforded by the computational schemes, especially for the $7 \mathrm{a}_{1}\left(\sigma_{\mathrm{N} L P}\right)^{-1}$ state, as discussed in Sec. IVC.

Obviously, the best estimates of the vertical ionization energies for the $7 a_{1}\left(\sigma_{N L P}\right)^{-1}$ and $1 \mathrm{a}_{2}(\pi)^{-1}$ states are provided by the present EOM-IP-CCSDT/cc-pVTZ calculations $(9.61$ and $9.73 \mathrm{eV}$, respectively). These values can be improved further by adding the $\Delta E_{\infty} \mathrm{CBS}$ correction (defined as $\Delta E_{\infty}=E_{\mathrm{V}}(\mathrm{cc}-\mathrm{pV} \infty \mathrm{Z})-E_{\mathrm{V}}(\mathrm{cc}-\mathrm{pVTZ})$ ) using the results given in Table IV), to obtain 9.72 and $9.85 \mathrm{eV}$, respectively. For comparison, Reineck et al ${ }^{30}$ reported vertical ionization energies of 9.66 and $9.85 \mathrm{eV}$ for the HOMO and HOMO-1 orbitals, from their HeI excited spectrum. Similar accurate theoretical results, of 9.54 and $9.65 \mathrm{eV}$, were obtained previously only in the CASPT2 study of Lorentzon et al. ${ }^{46}$ As can be seen from Table V, other theoretical methods giveless accurate results for the lowest two cationic states, and in some cases reverse the order established by the CCSDT benchmarks.

\section{Adiabatic ionization}

We have used the MP2 and CCSD geometries (Tables IX and X) to compute the adiabatic ionization energies and to evaluate the adiabatic energy corrections $\left(\Delta E_{\mathrm{ad}}\right)$ to the vertical ionization energies $\Delta E_{\mathrm{v}}$ (Table V). The $\Delta E_{\mathrm{ad}}$ corrections are obtained as the differences between the total energies $\left(E_{\text {tot }}\right)$ for the cationic state at its optimal geometry (CS) and the ground-state optimal geometry (GS): $\Delta E_{\mathrm{ad}}=E_{\mathrm{tot}}(\mathrm{CS})-E_{\mathrm{tot}}(\mathrm{GS})$. The final-state vibrational frequencies were computed at the MP2 level (Table XI), and the corresponding 00 corrections $\left(\Delta E_{0-0}\right)$ to the adiabatic ionization energies were evaluated (Table $\mathrm{V}$ ). The latter are defined as the difference between the zero-point vibrational energies (ZPVE) for the cationic state and the electronic ground state: $\Delta E_{0-0}=Z P V E(\mathrm{CS})-Z P V E(\mathrm{GS})$. The $\Delta E_{\mathrm{ad}}$ CCSD adiabatic and $\Delta E_{0-0} \mathrm{MP} 20-0$ corrections as well as the $\Delta E_{\infty}$ CBS correction were added to the EOM-IP-CCSDT vertical ionization energies to obtain the best estimates $\left(E_{0-0}\right)$ of the $0-0$ ionization energies (Table V). 
Our best estimate for the $7 \mathrm{a}_{1}\left(\sigma_{\mathrm{N} L P}\right)^{-1} 0-0$ transition energy is $9.31 \mathrm{eV}$. This energy is still slightly higher than the $E_{0-0}$ values, $9.199,{ }^{34} 9.197,{ }^{36} 9.1978,{ }^{38}$ and $9.1215,{ }^{39} \mathrm{eV}$, obtained in recent experiments. It is interesting to note that Riese et al. ${ }^{38}$ attempted to characterize the lowest cationic state using density functional theory with a B3LYP functional and the aug-ccpVTZ basis set. The resulting cationic ground state was predicted to be of ${ }^{2} B_{2}$ symmetry, with a molecular structure differing considerably from that obtained in our CCSD/cc-pVTZ treatment of the ${ }^{2} \mathrm{~B}_{2}$ state.

The best estimate for the $0-0$ transition energy of the $1 a_{2}(\pi)^{-1 /}$ state obtained in the present theoretical work is $9.65 \mathrm{eV}$. The corresponding experimental value is difficult to determine due to the complexity of the lowest photoelectron band, to which this transition contributes. The only experimental value, $9.275 \mathrm{eV}^{39}$, is much lower than our prediction. In view of the very small vertical gap between the $7 \mathrm{a}_{1}\left(\sigma_{\mathrm{N}} \mathrm{LP}\right)^{-1}$ and $1 \mathrm{a}_{2}(\pi)^{-1}$ states $(0.12-0.13 \mathrm{eV}$ at the EOM-IP-CCSDT level of theory), it is very likely that these two states are subject to strong vibronic coupling. This coupling probably also involves the $2 b_{1}(\pi)^{-1}$ state, which lies $0.7 \mathrm{eV}$ above the $1 \mathrm{a}_{2}(\pi)^{-1}$ state $($ Table $\mathrm{V})$. The characteristic appearance of the first two photoelectron bands observed in the HeI excited spectrum recorded by Liu et $\mathrm{al}^{34}$ supports this suggestion. Below the maximum (at $\sim 9.7 \mathrm{eV}$ ) of the first photoelectron band the spectrum is dominated by well-resolved vibrational excitations. These excitations become exceedingly dense near the band/maximum but disappear at binding energies above $\sim 10 \mathrm{eV}$. No vibrational structure is observed either on the high energy portion of the first photoelectron band or across the entire second photoelectron band, which occurs between 10.3 and $11.0 \mathrm{eV}$. Previous theoretical work has shown that resolved vibrational structure is usually absent above the point of a conical intersection between potential energy surfaces, as here the nuclear dynamics becomes strongly non-adiabatic and give rise to numerous irregular vibronic transitions. $^{101}$ (See, for example, Ref. 12 which shows photoelectron spectra of the dichlorobenzenes where vibronic coupling occurs between the lowest two ionic states). In pyridine it seems plausible to suggest that the lack of vibrational peaks above $\sim 10 \mathrm{eV}$ marks the presence of a conical intersection between the $7 a_{1}\left(\sigma_{N L P}\right)^{-1}$ and $1 a_{2}(\pi)^{-1}$ states. In such situations, accurate vertical and adiabatic transition energies can be extracted from 
experimental data only through using appropriate vibronic coupling models based on the results of high-level electronic structure methods. ${ }^{101,102}$

The second band in the photoelectron spectrum, with a maximum near $10.5 \mathrm{eV}$, can be attributed unambiguously to the $2 b_{1}(\pi)^{-1}$ final state. While the vertical energy of this transition is reproduced rather accurately by most of the theoretical methods (Tables III-V), the absence of any resolved vibrational structure in the experimental spectra indicates a more complex situation that can be understood only within the framework of a suitable vibronic coupling model. Our best estimate for the $2 b_{1}(\pi)$ ionization energy, $10.56 \mathrm{eV}$, obtained at the EOM-IP-CCSDT level of theory using CBS extrapolation, is in excellent agreement with the measured value (Table III). Our theoretical result for $\mathrm{E}_{0-0}, 10.30 \mathrm{eV}$, is also in accord with the $0-0$ transition energy of $10.315 \mathrm{eV}$ reported by Śmiałek et al. ${ }^{36}$

The next group of bands is located in the experimental spectrum between $\sim 12$ and $15 \mathrm{eV}$. The observed structure, due to four overlapping bands, is quite complex, with maxima occurring at $12.454,13.213 .8$ and $14.5 \mathrm{eV} .^{30}$ These features correspond to peaks $4-7$ in the theoretical spectrum (Figure 4), which, according to our calculations, can be assigned to ionization of the $5 b_{2}(\sigma), 1 b_{1}(\pi), 6 a_{1}(\sigma)$, and $5 b_{2}(\sigma)$ orbitals, respectively. The vertical EOMIP-CCSDT energy for ionization of the $5 \mathrm{~b}_{2}(\sigma)$ orbital, $12.88 \mathrm{eV}$ (CBS limit), is slightly higher than the measured value $\left(12.454 \mathrm{eV}^{30}\right)$. A shift in the same direction occurs for $\mathrm{E}_{0-0}$, where our calculated value of $12.31 \mathrm{eV}$ compares with the experimental result of $12.257 \mathrm{eV}{ }^{30}$ The vertical ionization energies, 13.27 and $14.04 \mathrm{eV}$, of the $1 \mathrm{~b}_{1}(\pi)$ and $6 \mathrm{a}_{1}(\sigma)$ orbitals, respectively, computed at the $\mathrm{ADC}(3)$ level, are in good agreement with the corresponding measurements (Table III).

Our calculations predict that satellites begin contributing to the spectrum above $14 \mathrm{eV}$. Several low-intensity $2 h-1 p$ transitions, including shake-up satellites associated with the $1 a_{2}(\pi), 5 b_{2}(\sigma)$, and $6 a_{1}(\sigma)$ main lines, appear in the theoretical spectrum (Figure 4$)$ at 14.16 , 14.28 and $14.79 \mathrm{eV}$. These satellites have relative intensities of $<0.01,0.02$, and 0.04 , respectively, compared to the corresponding main line. According to our ADC(3) results, the intensity of the $1 b_{2}(\pi)$ main line is 0.72 , which means that almost $30 \%$ of the intensity has 
been diverted to satellites. While not individually resolved, these satellites increase the width of the observed bands.

The next two bands in the experimental spectrum, with maxima at $15.838 \mathrm{eV}$ and $17.1 \mathrm{eV},{ }^{30}$ are relatively easy to assign. The first is due to ionization of the closely spaced $3 \mathrm{~b}_{2}(\sigma)$ and $5 \mathrm{a}_{1}(\sigma)$ orbitals, and the presence of these two contributions is reflected in the relatively high spectral intensity. ${ }^{30}$ The second band can be associated with the $4 \mathrm{a}_{1}(\sigma)$ orbital. Our calculations predict extensive satellite structure in the binding energy range encompassing the $4 a_{1}(\sigma)$ main line, so the observed width will be increased due to these unresolved contributions.

In common with many other organic compounds, the simple one-particle picture of ionization $^{4,61,62}$ becomes inappropriate at binding energies above $18 \mathrm{eV}$, as here a strong mixing of $1 h$ and $2 h-1 p$ configurations no longer allows a distinction between main lines and satellite states. The experimental spectrum of pyridine (Figure 4) contains a broad band between 19 and $21 \mathrm{eV}$, with a maximum at $\sim 19.5 \mathrm{eV}$. Our calculations suggest that this band is formed by three major components, exhibiting strong contributions from the $3 \mathrm{a}_{1}$ and $2 \mathrm{~b}_{2}$ orbitals, together with a large number of medium or low intensity $2 h$ - $1 p$-type transitions related to the $4 a_{1}, 3 a_{1}$, and $2 b_{2}$ orbitals.

The complexity of the photoelectron spectrum increases further towards higher binding energy. The broad band between 22 and $26 \mathrm{eV}$, with a maximum at $\sim 24 \mathrm{eV}$, and the band between 26 and $30 \mathrm{eV}$, with a maximum at $\sim 28 \mathrm{eV}$, are formed by various states reflecting a strong mixing between the $1 \mathrm{~b}_{2}, 2 \mathrm{a}_{1}$ and $1 \mathrm{a}_{1}$ hole states and a large number of $2 h-1 p$ configurations. As a result of this $1 h / 2 h-1 p$ mixing, the intensity of the individual final states decreases, while the number of final states grows. The spectral intensity originally allocated to a $1 \mathrm{~h}$ state is shared amongst a manifold of final states and there is no longer a dominant $1 \mathrm{~h}$ state. This situation is referred to as the "break-down of the orbital picture of ionization". 4 


\section{G. Photoelectron angular distributions and photoionization partial cross- sections}

Photoelectron angular distributions can often be used to help assign the type of orbital ( $\pi, \sigma$ or non-bonding) associated with a specific band even in congested regions of the spectrum where the individual bands partially overlap. Previous experimental ${ }^{61,103,104}$ and theoretical $^{12,41,105}$ work has established that anisotropy parameters due to $\pi, \sigma$ or non-bonding orbitals exhibit differing energy dependences, and that these differences are particularly evident in the photon energy range $\sim 40-60 \mathrm{eV}$. The $\beta$-parameter corresponding to ionization of a $\pi$-orbital tends to lie close to zero at threshold, and then rises rapidly as the photon energy increases to reach a plateau value of at least $1.5 \mathrm{at} \mathrm{hv} \sim 60 \mathrm{eV}$. In contrast, the value of an anisotropy parameter associated with a $\sigma$-orbital rises much more slowly as the energy increases. In favorable circumstances, these differing energy dependencies allow the type of orbital associated with a specific photoelectron band to be identified through simple comparison of photoelectron spectra recorded either parallel or perpendicular to the polarization vector of the incident plane polarized radiation.

Figure 5 shows an example where comparison of perpendicular and parallel photoelectron spectra enable the band due to a $\pi$-orbital to be distinguished from neighboring bands associated with $\sigma$-orbitals. The band due to the $1 b_{1}(\pi)$ orbital, whose maximum occurs around $13 \mathrm{eV}$, is much stronger in the spectrum recorded at $\theta=0^{\circ}$ than it is in that recorded at $\theta=90^{\circ}$, because, at a photon energy of $40 \mathrm{eV}$, the $\beta$-parameter of a $\pi$-orbital is much higher than that of a $\sigma$-orbital. Thus, the band due to the $1 b_{1}(\pi)$ orbital is readily distinguished from those due to the adjacent $5 b_{2}(\sigma), 6 a_{1}(\sigma)$ and $4 b_{2}(\sigma)$ orbitals. A full analysis of parallel and perpendicular recordings, extended across all measured bands, allows quantitative experimental anisotropy parameters to be derived as a function of photon energy from our angle resolved spectra.

In previous experimental studies ${ }^{29,31,34}$ the variation of the $\beta$-parameter, as a function of binding energy, for the outermost photoelectron band of pyridine, encompassing contributions from the $7 \mathrm{a}_{1}\left(\sigma_{\mathrm{NLP}}\right)$ and $1 \mathrm{a}_{2}(\pi)$ orbitals, has been used as an indication of the ordering of these two orbitals. These analyses suggest that the ionization energy of the $7 \mathrm{a}_{1}$ orbital is lower than that of the $1 \mathrm{a}_{2}$ orbital. Our present results do not allow such a definite conclusion on the 
orbital sequence to be reached. Although at some photon energies the value of the $\beta$ parameter for the low binding energy region of the band is lower than that for higher binding energies, in accord with earlier analyses, ${ }^{29,31}$ at other photon energies the opposite variation has been found. Moreover, at many photon energies the $\beta$-parameter does not appear to vary significantly across the band. Such a behavior would be consistent with that expected from two substantially overlapping bands whose ionization energies are similar, as our calculations predict, and as previously suggested by Liu et al. ${ }^{34}$ It should also be noted that at low photon energies, such as those used in earlier experiments, ${ }^{29,31}$ the ionization process may be influenced by autoionization, which is known to affect $\beta$-parameter values.

Figure 6 shows photoelectron anisotropy parameters for the $1 a_{2}(\pi), 7 a_{1}\left(\sigma_{N} L P\right), 2 b_{1}(\pi)$ and $5 b_{2}(\sigma)$ orbitals measured in the present experiment together with theoretical predictions. A complete set of experimental and theoretical $\beta$-parameters for all the energy regions specified in Table I is available in the Supplementary Material. In general the agreement between experiment and theory is reasonable and demonstrates the expected differing energy dependencies for ionization from a $\sigma$ - or $\pi$-orbital. The $\beta$-parameter for the $2 b_{1}(\pi)$ orbital rises rapidly with increasing energy (as shown by both experiment and theory) in contrast to that for the $5 b_{2}(\sigma)$ orbital where the increase in the $\beta$-value is more gradual. Indeed, this contrasting energy variation is evident in the theoretical predictions for the outermost band, comprising contributions from the $7 \mathrm{a}_{1}\left(\sigma_{\mathrm{N} L P}\right)$ and $1 \mathrm{a}_{2}(\pi)$ orbitals. The calculated $\beta$-parameter for the $1 a_{2}$ orbital increases more rapidly than that associated with the $7 a_{1}$ orbital, with the individual curves reaching similar values for a photon energy of $\sim 100 \mathrm{eV}$. These two contributions are not resolved experimentally and in the photon energy range $20-50 \mathrm{eV}$ the measured anisotropy parameter lies between those predicted for the $7 a_{1}$ and $1 a_{2}$ orbitals. It should be noted that the representation of the $7 a_{1}$ orbital in these calculations includes none of the post HF refinements discussed above.

The experimentally derived and the calculated absolute photoionization partial crosssections for the $1 a_{2}(\pi), 7 a_{1}\left(\sigma_{N} L P\right), 2 b_{1}(\pi)$ and $5 b_{2}(\sigma)$ orbitals are plotted in Figure 7 . Again, a complete set of results is available in Supplementary Material. The overall agreement between experiment and theory is satisfactory, especially at photon energies above $\sim 30 \mathrm{eV}$. For some 
Publishing orbitals, the prominent resonances predicted a few eV above threshold appear overestimated.

Such effects have been observed previously in fixed geometry CMS-X $\alpha$ calculations. ${ }^{106}$ The inclusion of vibrational averaging tends to both reduce the magnitude of the resonance and to increase the peak width.

\section{SUMMARY AND CONCLUSIONS}

The outer- and inner-valence shell ionization spectra of pyridine were investigated using the $\mathrm{ADC}(3)$ approximation scheme for the one-particle Green's function. The vertical ionization energies and the corresponding spectral intensities (pole strengths) were computed, and these were used to interpret the experimental photoelectron spectra. To limit the computational cost, the relatively compact cc-pVDZ basis sets were used in these calculations. These, nevertheless, allow a qualitatively correct description of the spectrum to be obtained.

Outer-valence ionization transitions were also computed using the OVGF and EOM-IPCCSD methods. Here, the larger aug-cc-pVTZ basis sets were used as these allow the outervalence ionization to be described in a quantitative manner. At the OVGF level, a basis set convergence study was carried out employing the cc-pVnZ and aug-cc-p VmZ $(n=\mathrm{D}, \mathrm{T}, \mathrm{Q}, 5$, and $m=\mathrm{D}, \mathrm{T}, \mathrm{Q})$ sets. CBS extrapolation results were obtained.

The theoretical results were found to be in good agreement with the experimental data across the entire binding energy range, and enabled the structure observed in the photoelectron spectra to be assigned. The outer-valence ionic states are well described within the one-electron picture of ionization. The lowest satellite $(2 h-1 p)$ states appear at about $14 \mathrm{eV}$, and acquire their intensity from the innermost $\pi$-orbital. Hence, the spectral intensity of the $\pi^{-1}$ main state is somewhat decreased, as is typical for heteroaromatic molecules. ${ }^{61}$ The ionization of the inner-valence orbitals is influenced by large electron correlation and orbital relaxation effects. These effects are reflected in our calculations in strong $1 h$ and $2 h-1 p$ configuration mixing and in the transfer of intensity from the main $(1 h)$ state to a manifold of final states. This situation, also referred to as the break-down of the one-electron picture of ionization, ${ }^{4}$ occurs in the spectra at binding energies above $20 \mathrm{eV}$. In the $\mathrm{ADC}(3)$ method, the 
$2 h-1 p$ states are treated less accurately (first order) than the $1 h$ states (third order), thereby affording only a qualitative description of the inner-valence spectral regime.

The effects of electron correlation and orbital relaxation can also be important for outervalence ionization. This is demonstrated by our study of the lowest four states, ${ }^{2} \mathrm{~A}_{2}\left(1 \mathrm{a}_{2}{ }^{-1}\right)$, ${ }^{2} \mathrm{~A}_{1}\left(7 \mathrm{a}_{1}^{-1}\right),{ }^{2} \mathrm{~B}_{1}\left(2 \mathrm{~b}_{1}{ }^{-1}\right)$, and ${ }^{2} \mathrm{~B}_{2}\left(5 \mathrm{~b}_{2}{ }^{-1}\right)$, of the pyridine radical cation. The relative positions of the corresponding energy levels, particularly that of the $7 a_{1}(n \sigma)$ orbital, has been a major issue in the theoretical and experimental literature. For a definitive clarification, we have used high-level electronic structure methods, including the EOM-IP-CCSDT scheme, together with the high-quality cc-pVTZ basis set and CBS limit extrapolation. According to our best estimates, the $7 a_{1}(n \sigma)^{-1}$ state is the ground state of the pyridine cation, with a vertical ionization energy of $9.72 \mathrm{eV}$, followed by the $1 \mathrm{a}_{2}(\pi)^{-1}$ state at $9.85 \mathrm{eV}$, the $2 b_{1}(\pi)^{-1}$ state at $10.56 \mathrm{eV}$, and the $5 \mathrm{~b}_{2}(\sigma)^{-1}$ state at $12.88 \mathrm{eV}$.

For each of the four lowest cationic states, the equilibrium geometry parameters were computed using the MP2 and CCSD methods, and the harmonic vibrational frequencies were obtained at the MP2 level. These results were used to estimate the adiabatic (0-0) ionization energies $(9.31,9.65,10.30,12.31 \mathrm{eV})$. Except for the $1 \mathrm{a}_{2}(\pi)^{-1}$ state, the theoretical $0-0$ estimates are in accord with the experimental data. For the $1 \mathrm{a}_{2}(\pi)^{-1}$ state, vibronic coupling to the lower $7 \mathrm{a}_{1}(\mathrm{n} \sigma)^{-1}$ state may play a role, as indicated by the small energy gap between the two states and the rather complex appearance of the $1 \mathrm{a}_{2}(\pi)$ photoelectron band. ${ }^{34}$

The orbital relaxation and electron correlation effects in the $7 a_{1}(n \sigma), 1 a_{2}(\pi), 2 b_{1}(\pi)$, and $5 b_{2}(\sigma)$ transitions were analyzed in detail. Often an ionic state is reasonably well described as a $1 h \mathrm{HF}$ configuration where the unaffected electrons reside in the ground-state (frozen) HF orbitals. That is the first-order Koopmans description of ionization. At the second-order level, electron correlation and orbital relaxation comes into play, and it is interesting to analyze the corresponding changes in the electron density in the ionic states. To this end, we have computed total electron density difference maps, allowing the frozen and relaxed densities to be compared.

A very strong orbital relaxation effect, amounting to $-2.73 \mathrm{eV}$, was found for the $7 a_{1}(n \sigma)^{-1}$ state. This is much larger than the corresponding values of $-0.85,-0.96$, and 
$-0.86 \mathrm{eV}$ for the three other low-lying cationic states, $1 \mathrm{a}_{2}(\pi)^{-1}, 2 \mathrm{~b}_{1}(\pi)^{-1}$, and $5 \mathrm{~b}_{2}(\sigma)^{-1}$, respectively. In contrast, the electron correlation contributions, $1.57,1.18,1.30$, and $0.81 \mathrm{eV}$, respectively, are more uniform. The exceptionally large relaxation shift for the nitrogen lonepair state poses an obvious challenge to the electronic structure methods used here.

An explicit PT2 expansion of the relaxation energy allowed the relaxation effect in the n $\sigma$ lone-pair state to be analyzed in detail. More specifically, by employing the PT2 approach, the main contributions to the relaxation energy in terms of two-electron integrals were identified. The screening of the n $\sigma$ positive charge is described by the $\left(\sigma \sigma \mid \pi \pi^{*}\right) \equiv\left\langle\pi\left|\hat{J}_{\sigma}\right| \pi^{*}\right\rangle$ integral reflecting the coupling of the $\sigma$ charge to the density in the highest $\pi$ and the lowest $\pi^{*}$ orbitals. According to our results, more than $50 \%$ of the relaxation energy of the n $\sigma$ orbital is due to the $\pi$-system contribution. This is in agreement with the density difference plots predicting for the no orbital considerable changes in the electron density distribution above the molecular plane. For comparison, the $\pi$-system contributes only about $20 \%$ to the relaxation of $5 b_{2}(\sigma)^{-1}$ state.

The relaxation mechanism discussed above seems to be rather general and can be present in other heteroaromatic molecules possessing $\pi$-systems and n $\sigma$-type lone-pairs of the heteroatoms (nitrogen or oxygen). It may be responsible for the near degeneracy between the no and $\pi$ levels noticed in previous works on such molecules. ${ }^{49-58}$ This will be checked in our future studies.

Photoelectron anisotropy parameters and photoionization partial cross-sections for the outer-valence orbitals have been measured from the ionization threshold to a photon energy of $120 \mathrm{eV}$. The experimental data have been compared to the corresponding theoretical predictions obtained with the CMS-X $\alpha$ approach. The overall agreement is satisfactory, thereby supporting the predicted electronic orbital configuration.

\section{SUPPLEMENTARY MATERIAL}

See supplementary material for contour plots of pyridine total electron density differences showing the effects of orbital relaxation and electron correlation for ionization from the $7 a_{1}\left(\sigma_{N} L P\right), 1 a_{2}(\pi), 2 b_{1}(\pi)$, and $5 b_{2}(\sigma)$ orbitals. Supplementary material also contains a 
complete set of experimental and theoretical $\beta$-parameters, and a complete set of experimental and theoretical absolute photoionization partial cross-sections for all the regions specified in Table I.

\section{ACKNOWLEDGMENTS}

A.B.T. gratefully acknowledges Grant No. 4.1671.2017/4.6 from the Ministry of education and science of the Russian Federation. The authors are indebted to D. Yu. Soshnikov for help with the electronic structure calculations. D.M.P.H. is grateful to the Science and Technology Facilities Council (United Kingdom) for financial support 


\section{REFERENCES}

[1] P. Kiuru and J. Yli-Kauhaluoma, in Heterocycles in natural product synthesis, edited by K. Majumdar and S. K. Chattopadhyay (Wiley-VCH Verlag GmbH \& Co. KGaA, Weinheim, 2011), p. 267.

[2] M. Baumann and I. R. Baxendale, Beilstein J. Org. Chem. 9, 2265 (2013).

[3] L. S. Cederbaum and W. Domcke, Adv. Chem. Phys. 36, 205 (1977).

[4] L. S. Cederbaum, W. Domcke, J. Schirmer, and W. von Niessen, Adv. Chem. Phys. 65, 115 (1986).

[5] J. Schirmer, L. S. Cederbaum, and O. Walter, Phys. Rev. A 28, 1237 (1983).

[6] J. Schirmer and G. Angonoa, J. Chem. Phys. 91, 1754 (1989).

[7] W. von Niessen, J. Schirmer, and L. S. Cederbaum, Comp. Phys. Reports 1, 57 (1984).

[8] H.-G. Weikert, H. -D. Meyer, L. S. Cederbaum, and F. Tarantelli, J. Chem. Phys. 104, 7122 (1996).

[9] J. Schirmer, A. B. Trofimov, and G. Stelter, J. Chem. Phys. 109, 4734 (1998).

[10] A. B. Trofimov and J. Schirmer, J. Chem. Phys. 123, 144115 (2005).

[11] M. Schneider, D. Yu. Soshnikov, D. M. P. Holland, I. Powis, E. Antonsson, M. Patanen, C. Nicolas, C. Miron, M. Wormit, A. Dreuw, and A. B. Trofimov, J. Chem. Phys. 143, 144103 (2015).

[12] D. M. P. Holland, I. Powis, A. B. Trofimov, I. L. Bodzuk, D. Yu. Soshnikov, A. W. Potts, and L. Karlsson, Chem. Phys. 448, 61 (2015); A. W. Potts, D. M. P. Holland, I. Powis, L. Karlsson, A. B. Trofimov, and I. L. Badsyuk, Chem. Phys. 415, 84 (2013); I. Pówis, A. B. Trofimov, I. L. Badsyuk, D. M. P. Holland, A. W. Potts, and L. Karlsson, Chem. Phys. 415, 291 (2013).

[13] F. Morini, B. Hajgató, and M. S. Deleuze, J. Phys. Chem. A 114, 9374 (2010).

[14] J. V. Ortiz, in Computational Chemistry: Reviews of Current Trends, edited by J. Leszcynski (World Scientific, Singapore, 1997), Vol. 2, p. 1.

[15] J. V. Ortiz, J. Chem. Phys. 108, 1008 (1998). 
This manuscript was accepted by J. Chem. Phys. Click here to see the version of record.

[16] V. G. Zakrzewski and W. von Niessen, J. Comput. Chem. 14, 13 (1993); V. G.

Zakrzewski and J. V. Ortiz, Int. J. Quantum Chem. 53, 583 (1995); V. G. Zakrzewski

and J. V. Ortiz, Int. J. Quantum Chem. S28, 23 (1994).

[17] J. V. Ortiz, J. Chem. Phys. 104, 7599 (1996).

[18] A. M. Ferreira, G. Seabra, O. Dolgounitcheva, V. G. Zakrzewski, and J. V. Ortiz, in Understanding Chemical Reactivity: Quantum-Mechanical Prediction Of

Thermochemical Data, edited by J. Cioslowski (Springer, Netherlands, 2001), Vol. 22, p. 131.

[19] H. Sekino and R. J. Bartlett, Int. J. Quantum. Chem., Symp. 18, 255 (1984); J. Geertsen, M. Rittby, and R. J. Bartlett, Chem. Phys. Lett. 164, 57 (1989); J. F. Stanton and R. J. Bartlett, J. Chem. Phys. 98, 7029 (1993); D. C. Comeau and R. J. Bartlett, Chem. Phys. Lett. 207, 414 (1993).

[20] A. I. Krylov, Ann. Rev. Phys. Chem. 59, 433 (2008); R. J. Bartlett, WIREs Comput. Mol. Sci. 2, 126 (2012).

[21] M. Nooijen and J. G. Snijders, Int. J. Quantum Chem., Symp. 26, 55 (1992); F. Stanton and J. Gauss, J. Chem. Phys. 101, 8938 (1994); M. Musial, S. A. Kucharski, and R. J. Bartlett, J. Chem. Phys. 118, 1128 (2003); P. U. Manohar, J. F. Stanton, and A. I. Krylov, J. Chem. Phys. 131, 114112 (2009).

[22] J. F. Stanton and J. Gauss, J. Chem. Phys. 103, 1064 (1995); M. Nooijen and J. G. Snijders, J. Chem. Phys. 102, 1681 (1995); A. K. Dutta, N. Vaval, and S. Pal, J. Chem. Theory Comput. 9, 4313 (2013); K. B. Bravaya, E. Epifanovsky, and A. I. Krylov, J. Phys. Chem. Lett. 3, 2726 (2012).

[23] H. Nakatsuji and K. Hirao, Chem. Phys. Lett. 47, 569 (1977); M. Ehara, Y. Ohtsuka, H. Nakatsuji, M. Takahashi, and Y. Udagawa, J. Chem. Phys. 122, 234319 (2005); M. Ehara, J. R. Gour, and P. Piecuch, Mol. Phys. 107, 871 (2009).

[24] K. K. Innes, I. G. Ross, and W. R. Moomaw, J. Mol. Spectrosc. 132, 492 (1988).

[25] A. D. Baker and D. W. Turner, Phil. Trans. Roy. Soc. Lond. A 268, 131 (1970).

[26] R. Gleiter, E. Heilbronner, and V. Hornung, Helv. Chim. Acta 55, 255 (1972).

[27] G. H. King, J. N. Murrell, and R. J. Suffolk, J. Chem. Soc. Dalton Trans. p564 (1972). 
[28] C. R. Brundle, M. B. Robin, and N. A. Kuebler, J. Am. Chem. Soc. 94, 1466 (1972).

[29] C. Utsunomiya, T. Kobayashi, and S. Nagakura, Bull. Chem. Soc. Jpn. 51, 3482 (1978).

[30] I. Reineck, R. Maripuu, H, Veenhuizen, L. Karlsson, K. Siegbahn, M. S. Powar, W. N. Zu, J. M. Rong, and S. H. Al-Shamma, J. Electron Spectrosc. Relat. Phenom. 27, 15 (1982).

[31] M. N. Piancastelli, P. R. Keller, J. W. Taylor, F. A. Grimm, and T. A. Carlson, J. Am. Chem. Soc. 105, 4235 (1983).

[32] J. O. Berg, D. H. Parker, and M. A. El-Sayed, Chem. Phys. Lett. 56, 411 (1978).

[33] N. Kishimoto and K. Ohno, J. Phys. Chem. A 104, 6940 (2000).

[34] S. -Y. Lui, K. Alnama, J. Matsumoto, K. Nishizawa, H. Kohguchi, Y. -P. Lee, and T. Suzuki, J. Phys. Chem. A 115, 2953 (2011).

[35] M. S. Moghaddam, A. D. O. Bawagan, K. H. Tan, and W. von Niessen, Chem. Phys. 207, 19 (1996).

[36] M. A. Śmiałek, M. A. MacDonald, S. Ptasińska, L. Zuin, and N. J. Mason, Eur. Phys. J. D 70, 42 (2016).

[37] M. Tsubouchi and T. Suzuki, J. Phys. Chem. A 107, 10897 (2003).

[38] M. Riese, Z. Altug, and J. Grotemeyer, Phys. Chem. Chem. Phys. 8, 4441 (2006).

[39] Y. R. Lee, D. W. Kang, H. L. Kim, and C. H. Kwon, J. Chem. Phys. 141, 174303 (2014).

[40] I. C. Walker, M. H. Palmer, and A. Hopkirk, Chem. Phys. 141, 365 (1989).

[41] Y. -I. Suzuki and T. Suzuki, J. Phys. Chem. A 112, 402 (2008).

[42] J. Wan, M. Hada, M. Ehara, and H. Nakatsuji, J. Chem. Phys. 114, 5117 (2001).

[43] O. Kitao and H. Nakatsuji, J. Chem. Phys. 88, 4913 (1988).

[44] O. Plashkevych, H. Ågren, L. Karlsson, and L. G. M. Pettersson, J. Electron Spectrosc. Relat. Phenom. 106, 51 (2000).

[45] L. Yang, H. Ågren, and V. Carravetta, J. Electron Spectrosc. Relat. Phenom. 87, 141 (1997)

[46] J. Lorentzon, M. P. Fülscher, and B. O. Roos, Theor. Chim. Acta 92, 67 (1995).

[47] W. von Niessen, W. P. Kraemer, and G. H. F. Diercksen, Chem. Phys. 41, 113 (1979). 
[48] W. von Niessen, G. H. F. Diercksen, and L. S. Cederbaum, Chem. Phys. 10, 345 (1975).

[49] O. Dolgounitcheva, V. G. Zakrzewski, and J. V. Ortiz, J. Phys. Chem. A 113, 14630 (2009).

[50] A. B. Trofimov, J. Schirmer, V. B. Kobychev, A. W. Potts, D. M. P. Holland, and L. Karlsson, J. Phys. B: At. Mol. Opt. Phys. 39, 305 (2006).

[51] K. B. Bravaya, O. Kostko, S. Dolgikh, A. Landau, M. Ahmed, and A. I. Krylov, J. Phys. Chem. A 114, 12305 (2010).

[52] A. W. Potts, D. M. P. Holland, A. B. Trofimov, J. Schirmer, L. Karlsson, and K. Siegbahn, J. Phys. B: At. Mol. Opt. Phys. 36, 3129 (2003).

[53] O. Dolgounitcheva, V. G. Zakrzewski, and J.V. Ortiz, J. Phys. Chem. A 106, 8411 (2002).

[54] D. M. P. Holland, A. W. Potts, L. Karlsson, I. L. Zaytseva, A. B. Trofimov, and J. Schirmer, Chem. Phys. 352, 205 (2008).

[55] D. M. P. Holland, A. W. Potts, L. Karlsson, I. L. Zaytseva, A. B. Trofimov, and J. Schirmer, Chem. Phys. 353, 47 (2008).

[56] O. Dolgounitcheva, V.G. Zakrzewski, and J. V. Ortiz, Int. J. Quant. Chem. 80, 831 (2000).

[57] I. L. Zaytseva, A. B. Trofimov, J. Schirmer, O. Plekan, V. Feyer, R. Richter, M. Coreno, and K. C. Prince, J. Phys. Chem. A 113, 15142 (2009).

[58] O. Dolgounitcheva, V. G. Zakrzewski, and J. V. Ortiz, J. Am. Chem. Soc. 122, 12304 (2000).

[59] A. L. Fetter and J. D. Walecka, Quantum Theory of Many-Particle Systems (McGrawHill, New York, 1971).

[60] A. A. Abrikosov, L. P. Gorkov, and I. E. Dzyaloshinski, Methods of Quantum Field Theory in Statistical Physics (Prentice-Hall, Englewood Cliffs, 1963).

[61] A. B. Trofimov, J. Schirmer, D. M. P. Holland, L. Karlsson, R. Maripuu, K. Siegbahn and A. W. Potts, Chem. Phys. 263, 167 (2001); A. W. Potts, A. B. Trofimov, J. Schirmer, D. M. P. Holland, and L. Karlsson, Chem. Phys. 271, 337 (2001). 
[62] I. Powis, I. L. Zaytseva, A. B. Trofimov, J. Schirmer, D. M. P. Holland, A. W. Potts, and L. Karlsson, J. Phys. B: At. Mol. Opt. Phys. 40, 2019 (2007).

[63] J. Schirmer and F. Mertins, Int. J. Quantum Chem. 58, 329 (1996).

[64] J. Lindeberg and Y. Öhrn, Propagators in Quantum Chemistry; $2^{\text {nd }}$ ed. (WileyInterscience, New Jersey, 2004).

[65] J. V. Ortiz, Adv. Quantum Chem. 35, 33 (1999).

[66] J. Schirmer, M. Braunstein, M.-T. Lee, and V. McKoy, in VUV and Soft X-Ray Photoionization, edited by U. Becker, D. A. Shirley (Plenum Press, New York, 2001), p. 105.

[67] C. M. Oana and A. I. Krylov, J. Chem. Phys.127, 234106 (2007); C. M. Oana and A. I. Krylov, J. Chem. Phys. 131, 124114 (2009)

[68] F. Morini, B. Hajgató, M. S. Deleuze, C. G. Ning, and J. K. Deng, J. Phys. Chem. A 112, 9083 (2008); M. S. Deleuze and S. Knippenberg, J. Chem. Phys. 125, 104309 (2006); S. Knippenberg, K. L. Nixon, H. Mackenzie-Ross, M. J. Brunger, F. Wang, M. S. Deleuze, J.-P. François, and D.A. Winkler, J. Phys. Chem. A 109, 9324 (2005).

[69] Y. R. Huang, S. Knippénberg, B. Hajgató, J.-P. François, J. K. Deng, and M. S. Deleuze, J. Phys. Chem. A 111, 5879 (2007).

[70] I. G. Kaplan, B. Barbiellini, and A. Bansil, Phys. Rev. B 68, 235104 (2003).

[71] E. Weigold and I. E. McCarthy, Electron Momentum Spectroscopy (Kluwer Dordecht/Plenum, New York, 1999).

[72] R. G. Parr and W. Yang, Density functional theory of atoms and molecules (Oxford University Press, New York, 1989).

[73] G. Born, H.A. Kurtz, and Y. Öhrn, J. Chem. Phys. 68, 74 (1978).

[74] The ADC(3) code originally written by G. Angonoa, O. Walter and J. Schirmer; further developed by M. K. Scheller and A. B. Trofimov.

[75] M. W. Schmidt, K. K. Baldridge, J. A. Boatz, S. T. Elbert, M. S. Gordon, J. H. Jensen, S. Koseki, N. Matsunaga, K. A. Nguyen, S. Su, T. L. Windus, M. Dupuis, and J. A. Montgomery, J. Comp. Chem. 14, 1347 (1993); M. S. Gordon and M. W. Schmidt, in Advances in electronic structure theory: GAMESS a decade later, edited by C . E. 
Dykstra, G. Frenking, K. S. Kim, and G. E. Scuseria, (Elsevier, Amsterdam, 2005), p. 1167.

[76] T. H. Dunning, J. Chem. Phys. 90, 1007 (1989); R. A. Kendall, T. H. Dunning, and R. J. Harrison, J. Chem. Phys. 96, 6769 (1992).

[77] M. J. Frisch, G. W. Trucks, H. B. Schlegel, G. E. Scuseria, M. A. Robb, J. R. Cheeseman, G. Scalmani, V. Barone, B. Mennucci, G. A. Petersson, H. Nakatsuji, M. Caricato, X. Li, H. P. Hratchian, A. F. Izmaylov, J. Bloino, G. Zheng, J. L. Sonnenberg, M. Hada, M. Ehara, K. Toyota, R. Fukuda, J. Hasegawa, M. Ishida, T. Nakajima, Y. Honda, O. Kitao, H. Nakai, T. Vreven, J. A. Montgomery, Jr., J. E. Peralta, F. Ogliaro, M. Bearpark, J. J. Heyd, E. Brothers, K. N. Kudin, V. N. Staroverov, T. Keith, R. Kobayashi, J. Normand, K. Raghavachari, A. Rendell, J. C. Burant, S. S. Iyengar, J. Tomasi, M. Cossi, N. Rega, J. M. Millam, M. Klene, J. E. Knox, J. B. Cross, V. Bakken, C. Adamo, J. Jaramillo, R. Gomperts, R. E. Stratmann, O. Yazyev, A. J. Austin, R. Cammi, C. Pomelli, J. W. Ochterski, R. L. Martin, K. Morokuma, V. G. Zakrzewski, G. A. Voth, P. Salvador, J. J. Dannenberg, S. Dapprich, A. D. Daniels, O. Farkas, J. B. Foresman, J. V. Ortiz, J. Cioslowski, and D. J. Fox, Gaussian 09, Revision C.01, Gaussian, Inc., Wallingford, CT, 2010.

[78] D. Feller, J. Chem. Phys. 96, 6104 (1992).

[79] T. Helgaker, W. Klopper, H. Koch, and J. Noga, J. Chem. Phys. 106, 9639 (1997).

[80] J. K. Min, E. C. Lee, H. M. Lee, D. Y. Kim, D. Kim, and K. S. Kim, Comput. Chem. 29, 1208 (2008).

[81] Y. Shao et al, Mol. Phys. 113, 184 (2015).

[82] J. F. Stanton and J. Gauss, J. Chem. Phys. 111, 8785 (1999).

[83] CFOUR, Coupled cluster techniques for Computational Chemistry, a quantum-chemical program package by J. F. Stanton, J. Gauss, M. E. Harding, and P. G. Szalay, with contributions from A. A. Auer, R. J. Bartlett, U. Benedikt, C. Berger, D. E. Bernholdt, Y. J. Bomble, L. Cheng, O. Christiansen, M. Heckert, O. Heun, C. Huber, T.-C. Jagau, D. Jonsson, J. Jusélius, K. Klein, W. J. Lauderdale, D. A. Matthews, T. Metzroth, L. A. Mück, D. P. O’Neill, D. R. Price, E. Prochnow, C. Puzzarini, K. Ruud, F. Schiffmann, 
W. Schwalbach, C. Simmons, S. Stopkowicz, A. Tajti, J. Vázquez, F. Wang, and J. D. Watts and the integral packages MOLECULE (J. Almlöf and P. R. Taylor), PROPS (P. R. Taylor), ABACUS (T. Helgaker, H. J. Aa. Jensen, P. Jørgensen, and J. Olsen), and ECP routines by A. V. Mitin and C. van Wüllen. For the current version, see http://www.cfour.de.

[84] R. Ditchfield, W. J. Hehre, and J. A. Pople, J. Chem. Phys. 54, 724 (1971); W. J. Hehre, R.Ditchfield and J. A. Pople, J. Chem. Phys. 56, 2257 (1972); P. C. Hariharan and J. A. Pople, Theoret. Chimica Acta 28, 213 (1973).

[85] GaussView, Version 5, R. Dennington, T. Keith, and J. Millam, Semichem Inc., Shawnee Mission, KS, 2009.

[86] G. Schaftenaar and J. H. Noordik, "Molden: a pre- and post-processing program for molecular and electronic structures", J. Comput.-Aided Mol. Design 14, 123 (2000).

[87] D. M. P. Holland, I Powis, G. Öhrwall, L. Karlsson, and W. von Niessen, Chem. Phys. 326, 535 (2006).

[88] Y. Hikosaka, J. H. D. Eland, T. M. Watson, and I. Powis, J. Chem. Phys. 115, 4593 (2001).

[89] D. M. P. Holland, Phys. Scripta 36, 22 (1987).

[90] P. Finetti, D. M. P. Holland, C. J. Latimer, C. Binns, F. M. Quinn, M. A Bowler, A. F. Grant, and C. S. Mythen, Nucl. Instrum. Methods Phys. Res. B 184 627, (2001).

[91] D. M. P. Holland, M. A. Mac Donald, M. A. Hayes, P. Baltzer, L. Karlsson, M. Lundqvist, B. Wannberg, and W. von Niessen, Chem. Phys. 188, 317 (1994).

[92] S. H. Southworth, A. C. Parr, J. E. Hardis, J. L. Dehmer, and D. M. P. Holland, Nucl. Instrum. Methods Phys Res. A 246, 782 (1986).

[93] S. Tixier, G. Cooper, R. Feng, and C. E. Brion, J. Electron Spectrosc. Relat. Phenom. 123,185 (2002).

[94] L-C. Wang and R. J. Boyd, J. Chem. Phys. 90, 1083 (1989); R. J. Boyd and L-C. Wang, J. Comp. Chem. 10, 367 (1989).

[95] G. J. M. Veldem and D. Feil, J. Phys. Chem. 96, 10725 (1992). 

Struct. 156, 315 (1987).

[97] D. P. DiLella and H. D. Stidham, J. Raman Spectrosc. 9, 90 (1980).

[98] A. Langseth and R. C. Lord, Kgl. Danske Videnskab. Selskab, Mat.-fys. Medd. 16, 6 (1938).

[99] G. Zerbi, B. Crawford, Jr., and J. Overend, J. Chem. Phys. 38, 127 (1963).

[100] M. Kumar, M. Srivastava, and R.A. Yadav, Spectrochim. Aeta A: Molec. Biomolec. Spectrosc. 111, 242 (2013).

[101] H. Köppel, W. Domcke, and L. S. Cederbaum, Adv. Chem. Phys. 57, 59 (1984).

[102] A. B. Trofimov, H. Köppel, and J. Schirmer, J. Chem. Phys. 109, 1025 (1998).

[103] D. M. P. Holland, L. Karlsson, and W. von Niessen, J. Electron Spectrosc. Relat. Phenom. 113, 221 (2001).

[104] D. M. P. Holland, D. Edvardsson, L. Karlsson, R. Maripuu, K. Siegbahn, A. W. Potts, and W. von Niessen, Chem. Phys. 253, 133 (2000).

[105] D. M. P. Holland, A. W. Potts, L. Karlsson, M. Stener, and P. Decleva, Chem. Phys. 390, 25 (2011).

[106] J. L. Dehmer, D. Dill, and S. Wallace, Phys. Rev. Lett. 43, 1005 (1979). 


\section{TABLES}

Table I. Binding energy regions used in the analysis of the angle resolved photoelectron spectra.

\begin{tabular}{|c|c|c|}
\hline Region & Binding energy range $(\mathrm{eV})$ & Encompassed orbitals \\
\hline 1 & $8.7-10.2$ & $1 \mathrm{a}_{2}(\pi), 7 \mathrm{a}_{1}\left(\sigma_{\mathrm{NLP}}\right)$ \\
\hline 2 & $10.2-11.4$ & $2 \mathrm{~b}_{1}(\pi)$ \\
\hline 3 & $11.9-12.75$ & $5 \mathrm{~b}_{2}(\sigma)$ \\
\hline 4 & $12.75-13.5$ & $1 \mathrm{~b}_{1}(\pi)$ \\
\hline 5 & $13.5-14.0$ & $6 \mathrm{a}_{1}(\sigma)$ \\
\hline 6 & $14.0-15.15$ & $4 \mathrm{~b}_{2}(\sigma)$ \\
\hline 7 & $15.15-16.5$ & $5 \mathrm{a}_{1}(\sigma), 3 \mathrm{~b}_{2}(\sigma)$ \\
\hline 8 & $16.5-18.4$ & $\left(4 \mathrm{a}_{1}(\sigma)\right.$ \\
\hline 9 & $18.4-21.4$ & $3 \mathrm{a}_{1}(\sigma), 2 \mathrm{~b}_{2}(\sigma)$ \\
\hline 10 & $21.4-34.0$ & $1 \mathrm{~b}_{2}(\sigma), 2 \mathrm{a}_{1}(\sigma), 1 \mathrm{a}_{1}(\sigma)$ \\
\hline
\end{tabular}


Table II. Mulliken atomic population in the outer-valence molecular orbitals of pyridine (units are electrons; sum over all atoms is 2) calculated at the HF/cc-pVDZ level.

\begin{tabular}{|c|c|c|c|c|c|c|c|c|c|c|}
\hline Atom & $\begin{array}{c}7 \mathrm{a}_{1} \\
\left(\sigma_{\mathrm{N} \mathrm{LP}}\right)\end{array}$ & $\begin{array}{c}1 \mathrm{a}_{2} \\
(\pi)\end{array}$ & $\begin{array}{c}2 \mathrm{~b}_{1} \\
(\pi)\end{array}$ & $\begin{array}{c}5 \mathrm{~b}_{2} \\
(\sigma)\end{array}$ & $\begin{array}{c}1 \mathrm{~b}_{1} \\
(\pi)\end{array}$ & $\begin{array}{c}6 \mathrm{a}_{1} \\
(\sigma)\end{array}$ & $\begin{array}{c}4 \mathrm{~b}_{2} \\
(\sigma)\end{array}$ & $\begin{array}{c}5 \mathrm{a}_{1} \\
(\sigma)\end{array}$ & $\begin{array}{c}3 \mathrm{~b}_{2} \\
(\sigma)\end{array}$ & $\begin{array}{c}4 \mathrm{a}_{1} \\
(\sigma)\end{array}$ \\
\hline $\mathrm{N}$ & 1.24 & 0.01 & 0.59 & 0.16 & 0.53 & 0.34 & 0.02 & 0.05 & 0.36 & 0.14 \\
\hline $\mathrm{C}_{2}$ & 0.15 & 0.47 & 0.09 & 0.20 & 0.37 & 0.14 & 0.31 & 0.16 & 0.31 & 0.26 \\
\hline $\mathrm{C}_{3}$ & 0.12 & 0.52 & 0.27 & 0.25 & 0.25 & 0.12 & 0.31 & 0.32 & 0.33 & 0.19 \\
\hline $\mathrm{C}_{4}$ & 0.03 & 0.02 & 0.68 & 0.29 & 0.22 & 0.58 & 0.03 & 0.07 & 0.32 & 0.31 \\
\hline
\end{tabular}

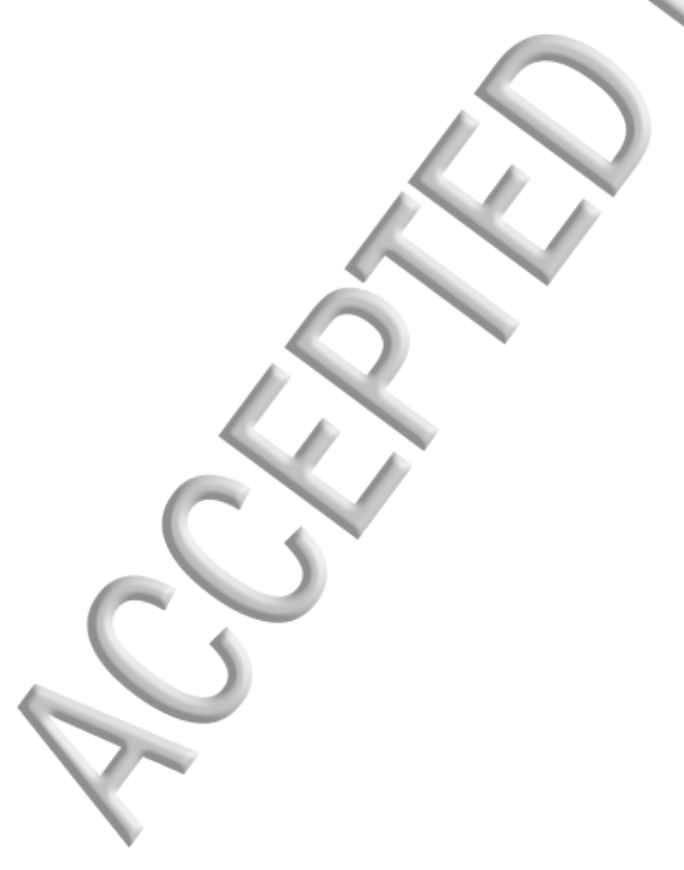


Table III. Energies $E(\mathrm{eV})$ and intensities $P$ of the outer-valence vertical ionization transitions in pyridine calculated using the HF, OVGF, ADC(3), and EOM-IP-CCSD methods and the cc-pVDZ and aug-cc-pVTZ basis sets. The experimental values are also listed.

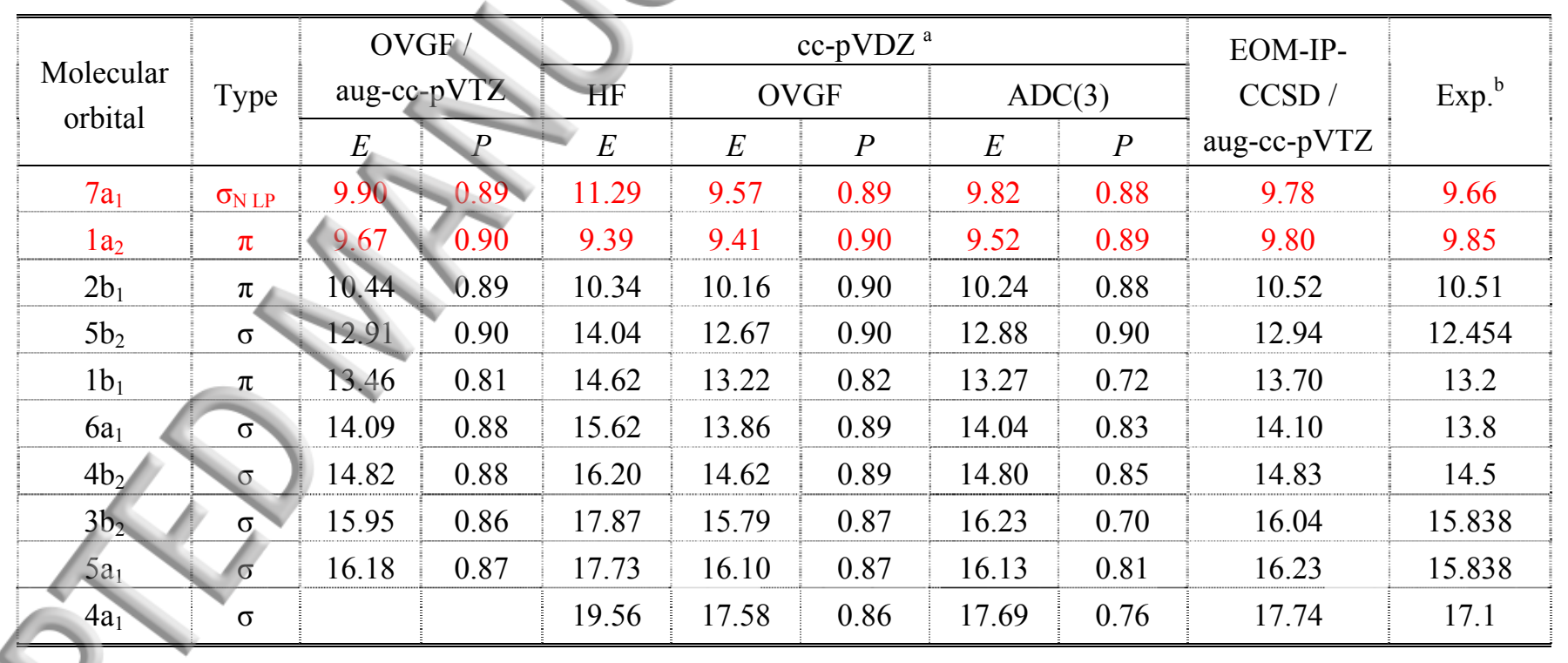

${ }^{a}$ Cartesian representation of d-function was used.

beak maxima reported by Reineck et al. ${ }^{30}$ 
Table IV. Vertical ionization energies of pyridine (eV) calculated at the OVGF level of theory using the series of basis sets cc-pVnZ and aug-cc-pVnZ with improving quality $(n=$ $\mathrm{D}, \mathrm{T}, \mathrm{Q}, 5)$, and results for the complete basis set (CBS) limits $(n \rightarrow \infty)$.

\begin{tabular}{|c|c|c|c|c|c|c|c|}
\hline Basis set & $7 \mathrm{a}_{1}$ & $1 \mathrm{a}_{2}$ & $2 \mathrm{~b}_{1}$ & $5 \mathrm{~b}_{2}$ & $1 \mathrm{~b}_{1}$ & $6 \mathrm{a}_{1}$ & $4 \mathrm{~b}_{2}$ \\
\hline cc-pVDZ & 9.58 & 9.42 & 10.17 & 12.68 & 13.24 & 13.87 & 14.63 \\
\hline aug-cc-pVDZ & 9.85 & 9.60 & 10.29 & 12.88 & 13.46 & 14.07 & 14.81 \\
\hline cc-pVTZ & 9.80 & 9.60 & 10.36 & 12.83 & 13.37 & 14.01 & 14.75 \\
\hline aug-cc-pVTZ & 9.90 & 9.67 & 10.44 & 12.91 & 13.46 & 14.09 & 14.82 \\
\hline cc-pVQZ & 9.87 & 9.67 & 10.44 & 12.89 & 13.42 & 14.07 & 14.80 \\
\hline aug-cc-pVQZ & 9.91 & 9.70 & 10.47 & 12.92 & 13.46 & 14.10 & 14.83 \\
\hline cc-pV5Z & 9.90 & 9.70 & 10.47 & 12.91 & 13.44 & 14.09 & 14.82 \\
\hline cc-pV $\infty Z$ & 9.91 & 9.72 & 10.49 & 12.93 & 13.45 & 14.11 & 14.83 \\
\hline aug-cc-pV $\infty Z$ & 9.91 & 9.72 & 10.48 & 12.93 & 13.46 & 14.11 & 14.83 \\
\hline \hline
\end{tabular}


Table V. Energies, $E_{\mathrm{v}}$, of the four lowest vertical ionization transitions of pyridine obtained using various computational schemes and the cc-pVTZ basis set in comparison with the previous results; complete basis set (CBS) correction, $\Delta E_{\infty}$; MP2 and CCSD adiabatic corrections to the vertical ionization energies, $\Delta E_{\mathrm{ad}}$; MP2 $0-0$ correction to the adiabatic ionization energies, $\Delta E_{0-0}$; the best estimates for $0-0$ ionization energies, $E_{0-0}$. All values are in $\mathrm{eV}$.

\begin{tabular}{|c|c|c|c|c|}
\hline & $7 \mathrm{a}_{1}\left(\sigma_{\mathrm{N} L P}\right)$ & $1 \mathrm{a}_{2}(\pi)$ & $2 \mathrm{~b}_{1}(\pi)$ & $5 b_{2}(\sigma)$ \\
\hline RHF/Koopmans' & 11.37 & 9.44 & 10.42 & 14.10 \\
\hline $\mathrm{RHF} / \triangle \mathrm{SCF}$ & 8.64 & 8.59 & & 13.24 \\
\hline $\mathrm{UHF} / \triangle \mathrm{SCF}^{\mathrm{a}}$ & 8.34 & 8.17 & & 12.89 \\
\hline$\triangle \mathrm{MP} 2$ & 10.73 & 10.19 & 11.43 & 14.27 \\
\hline$\triangle \mathrm{MP3}$ & 10.22 & 9.78 & 10.79 & 14.04 \\
\hline$\triangle \mathrm{MP4}$ & 10.33 & 9.95 & 10.99 & 13.74 \\
\hline$\Delta \mathrm{CCD}$ & 10.21 & 9.77 & 10.76 & 14.05 \\
\hline$\triangle \mathrm{CCSD}$ & 9.67 & 9.63 & 10.38 & 13.18 \\
\hline$\Delta \operatorname{CCSD}(\mathrm{T})$ & 9.71 & 9.76 & 10.51 & 12.92 \\
\hline OVGF & 9.80 & 9.60 & 10.36 & 12.83 \\
\hline $\mathrm{ADC}(3)$ & 10.06 & 9.65 & 10.39 & 13.06 \\
\hline EOM-IP-CCSD & 9.69 & 9.72 & 10.43 & 12.87 \\
\hline EOM-EE-CC $3^{b}$ & & 9.70 & 10.41 & 12.76 \\
\hline EOM-IP-CCSDT & 9.61 & 9.73 & 10.43 & 12.78 \\
\hline $\mathrm{EOM}^{-I P-C C S D T}{ }_{\infty}^{\mathrm{c}}$ & 9.72 & 9.85 & 10.56 & 12.88 \\
\hline $\operatorname{ADC}(3)^{d}$ & 9.78 & 9.44 & 10.15 & 12.73 \\
\hline $\mathrm{SAC}-\mathrm{CI}^{\mathrm{e}}$ & 9.23 & 9.36 & 10.12 & 12.48 \\
\hline CASPT $2^{f}$ & 9.54 & 9.65 & 10.37 & \\
\hline & 8.84 & 9.53 & 10.13 & 12.91 \\
\hline$\Delta E_{0}(\mathrm{OVGF})^{\mathrm{h}}$ & 0.11 & 0.12 & 0.13 & 0.10 \\
\hline$\Delta E_{\mathrm{ad}}(\mathrm{MP} 2)^{\mathrm{i}}$ & -0.66 & -0.16 & $-0.30^{\mathrm{j}}$ & \\
\hline$\Delta E_{\text {ad }}(\mathrm{CCSD})^{\mathrm{i}}$ & -0.52 & -0.16 & -0.18 & -0.57 \\
\hline$\Delta E_{0-0}(\mathrm{MP} 2)^{\mathrm{k}}$ & 0.11 & -0.04 & $-0.08^{\mathrm{j}}$ & \\
\hline$E_{0-0}{ }^{1}$ & 9.31 & 9.65 & 10.30 & 12.31 \\
\hline
\end{tabular}

${ }^{a}$ The calculated $\mathrm{S}^{2}$ values are $0.89,1.22,1.12$, and 1.35 for the $1 \mathrm{a}_{2}^{-1}(\pi), 7 \mathrm{a}_{1}{ }^{-1}\left(\sigma_{\mathrm{N} \mathrm{LP}}\right)$, $2 \mathrm{~b}_{1}^{-1}(\pi)$, and $5 \mathrm{~b}_{2}^{-1}(\sigma)$ states, respectively.

${ }^{\mathrm{b}}$ EOM-EE-CC3 method with continuum orbital approximation. ${ }^{82}$

${ }^{\mathrm{c}}$ Estimated CBS limit obtained by adding the $\Delta E_{\infty}$ correction to the EOM-IP-CCSDT/ccpVTZ result.

${ }^{\mathrm{d}}$ Ref. 35. ${ }^{\mathrm{e}}$ Ref. $42 .{ }^{\mathrm{f}}$ Ref. $46 .{ }^{\mathrm{g}}$ Ref. 40.

${ }^{\mathrm{h}}$ See text for details (Sec. IV.F.1). 
${ }^{\mathrm{i}}$ See text for details (Sec. IV.F.2).

${ }^{\mathrm{j}} \mathrm{C}_{2 \mathrm{v}}$ molecular structure (saddle point, as indicated by the two imaginary frequencies).

${ }^{\mathrm{k}}$ See text for details (Sec. IV.F.2).

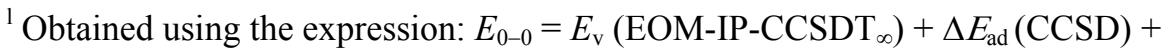
$\Delta E_{0-0}(\mathrm{MP} 2)$. For the $5 \mathrm{~b}_{2}(\sigma)$ transition the $\Delta E_{0-0}$ (MP2) term is omitted, since the MP2 geometry optimization failed for the final state.

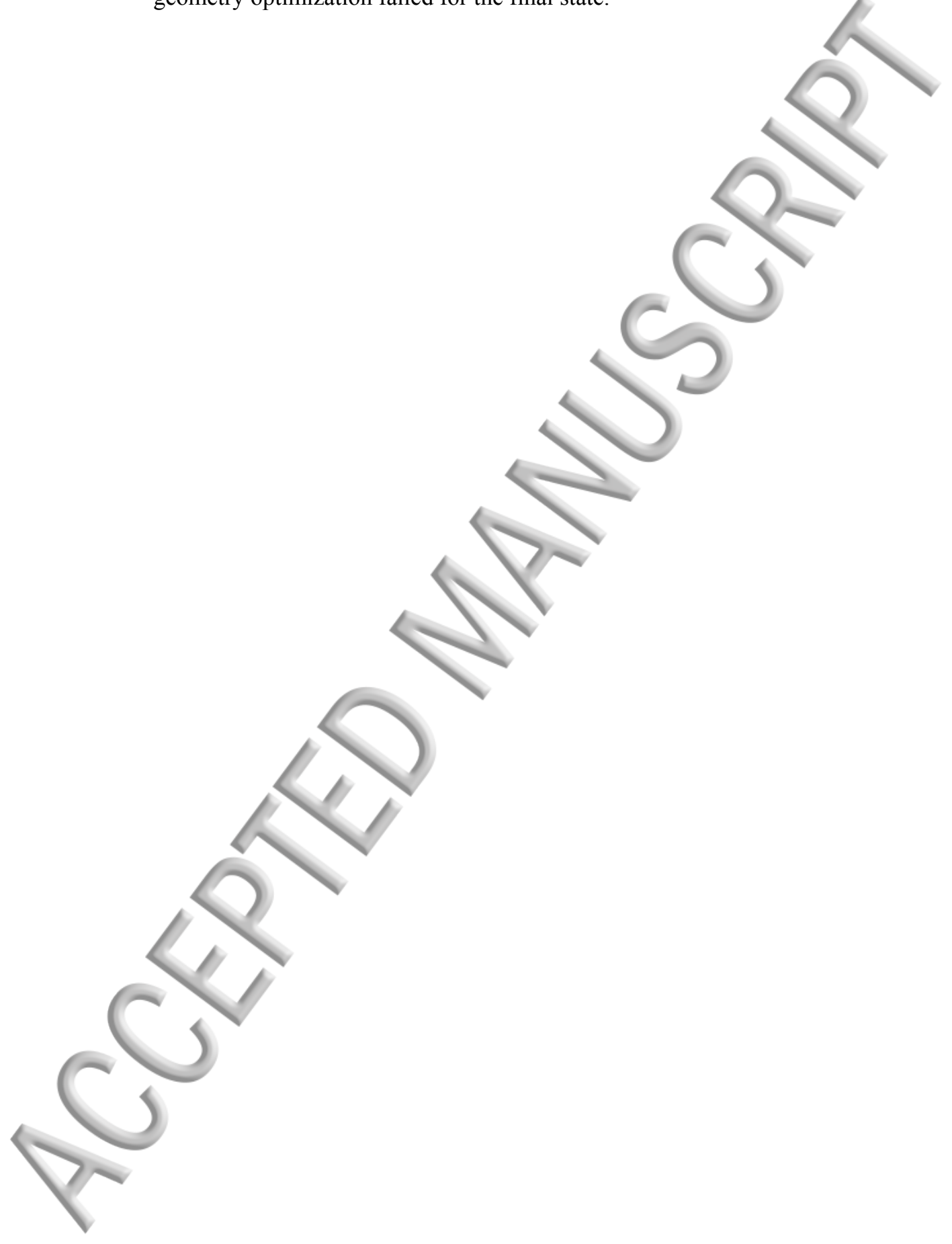


Table VI. Comparison of Mulliken atomic population in the four highest occupied molecular orbitals of pyridine in its singlet electronic ground state and the pyridine cation radicals in doublet electronic states obtained by ionization of the respective MOs (units are percent of the total orbital populations, equal to two and one, respectively). Calculations performed at the RHF/ccpVDZ level of theory

\begin{tabular}{|c|c|c|c|c|c|c|c|c|}
\hline \multicolumn{9}{|c|}{ Population in molecular orbitals } \\
\hline & \multicolumn{4}{|c|}{$\begin{array}{l}\text { Population in } \\
\text { Neutral ground state }\end{array}$} & \multicolumn{4}{|c|}{ Cation radicals } \\
\hline & $\left(\sigma_{N L P}\right)$ & $\begin{array}{l}1 \mathrm{a}_{2} \\
(\pi)\end{array}$ & $\begin{array}{l}2 b_{1} \\
(\pi)\end{array}$ & $\begin{array}{l}5 \mathrm{~b}_{2} \\
(\sigma)\end{array}$ & $\begin{array}{c}7 \mathrm{a}_{1} \\
\left(\sigma_{\mathrm{NLP}}\right)\end{array}$ & $\begin{array}{l}1 \mathrm{a}_{2} \\
(\pi)\end{array}$ & $\begin{array}{l}2 b_{1} \\
(\pi)\end{array}$ & $\begin{array}{l}5 \mathrm{~b}_{2} \\
(\sigma)\end{array}$ \\
\hline $\mathrm{N}$ & 62.0 & 0.5 & 29.5 & 8.0 & 88.0 & 0.0 & 29.0 & 3.0 \\
\hline & 7.5 & 23.5 & 4.5 & 10.0 & 3.0 & 23.0 & 4.0 & 3.0 \\
\hline $\mathrm{C}_{3}$ & 6.0 & 26.0 & 13.5 & 12.5 & 2.0 & 26.0 & 12.0 & 21.0 \\
\hline $\mathrm{C}_{4}$ & 1.5 & 1.0 & 34.0 & 14.5 & 0.0 & 1.0 & 40.0 & 27.0 \\
\hline All ring atoms & 91 & 100 & 100 & 68 & 98 & 100 & 100 & 78 \\
\hline
\end{tabular}


Table VII. Relaxation energies, $\Delta E^{R}(\mathrm{eV})$, and ionization potentials, IP $(\mathrm{eV})$, for the highest occupied orbitals of pyridine, computed using the second-order perturbation-theoretical (PT2) approach and the $\triangle \mathrm{SCF}$ method. Contribution of the $\pi$-type orbitals to the total relaxation energy, $\Delta E_{\pi}^{R}(\mathrm{eV})$. The percent of $\Delta E_{\pi}^{R}$ in the total $\Delta E^{R}$ is shown in parentheses. The HF IPs are also given for comparison. Calculations performed using the 6-31G* basis set.

\begin{tabular}{|l|c|c|c|c|}
\hline Quantity & $\begin{array}{c}7 \mathrm{a}_{1} \\
\left(\sigma_{\mathrm{N} \mathrm{LP}}\right)\end{array}$ & $\begin{array}{c}1 \mathrm{a}_{2} \\
(\pi)\end{array}$ & $\begin{array}{c}2 \mathrm{~b}_{1} \\
(\pi)\end{array}$ & $\begin{array}{c}5 \mathrm{~b}_{2} \\
(\sigma)\end{array}$ \\
\hline$\Delta E^{R}(\mathrm{PT} 2)$ & 2.54 & 0.98 & 1.07 & 0.67 \\
\hline$\Delta E^{R}(\Delta \mathrm{SCF})$ & 2.63 & 0.77 & 0.86 & 0.77 \\
\hline $\mathrm{IP}(\mathrm{PT} 2)$ & & & & \\
\hline $\mathrm{IP}(\Delta \mathrm{SCF})$ & 8.70 & 8.35 & 9.22 & 13.36 \\
\hline $\mathrm{IP}(\mathrm{HF})$ & 11.23 & 9.56 & 9.42 & 13.27 \\
\hline & & 9.33 & 10.28 & 14.03 \\
\hline$\Delta E_{\pi}^{R}(\mathrm{PT} 2)$ & $1.38(54)$ & $0.11(11)$ & $0.11(11)$ & $0.19(28)$ \\
\hline \hline
\end{tabular}


Table VIII. Five largest terms $S(k, i a)$ in the second-order perturbation-theoretical expansion of the relaxation energy (Eq. (4)) for the highest occupied orbitals $k$ of pyridine. The dominant $S_{1}$ terms together with the $(k k \mid a i)$ and $\left(\varepsilon_{a}-\varepsilon_{i}\right)$ quantities entering their nominator and denominator (Eq. (6)). All quantities are in $\mathrm{eV}$; calculations performed using the $6-31 \mathrm{G}^{*}$ basis set.

\begin{tabular}{|c|c|c|c|c|c|c|}
\hline$k$ & $i$ & $a$ & $S(k, i a)$ & $S_{1}(k, i a)$ & $(k k \mid a i)$ & $\left(\varepsilon_{a}-\varepsilon_{i}\right)$ \\
\hline \multirow{5}{*}{$7 a_{1}\left(\sigma_{N L P}\right)$} & $2 b_{1}(\pi)$ & $3 b_{1}\left(\pi^{*}\right)$ & 0.598 & 0.636 & 2.080 & 13.61 \\
\hline & $1 \mathrm{a}_{2}(\pi)$ & $2 \mathrm{a}_{2}\left(\pi^{*}\right)$ & 0.182 & 0.183 & -1.096 & 13.12 \\
\hline & $1 b_{1}(\pi)$ & $3 b_{1}\left(\pi^{*}\right)$ & 0.134 & 0.144 & 1.137 & 17.89 \\
\hline & $3 b_{2}(\sigma)$ & $9 b_{2}\left(\sigma^{*}\right)$ & 0.107 & 0.102 & -1.271 & 31.60 \\
\hline & $2 b_{1}(\pi)$ & $4 b_{1}\left(\pi^{*}\right)$ & 0.105 & 0.115 & 1.071 & 19.90 \\
\hline \multirow[t]{5}{*}{$1 a_{2}(\pi)$} & $4 b_{2}(\sigma)$ & $6 b_{2}\left(\sigma^{*}\right)$ & 0.127 & 0.130 & -1.248 & 23.97 \\
\hline & $4 a_{1}(\sigma)$ & $8 \mathrm{a}_{1}\left(\sigma^{*}\right)$ & 0.084 & 0.086 & -1.058 & 25.99 \\
\hline & $5 \mathrm{a}_{1}(\sigma)$ & $10 \mathrm{a}_{1}\left(\sigma^{*}\right)$ & 0.058 & 0.062 & -0.907 & 26.50 \\
\hline & $5 b_{2}(\sigma)$ & $7 b_{2}\left(\sigma^{*}\right)$ & 0.054 & 0.055 & 0.795 & 22.91 \\
\hline & $6 \mathrm{a}_{1}(\sigma)$ & $9 a_{1}\left(\sigma^{*}\right)$ & & 0.042 & 0.710 & 23.82 \\
\hline \multirow[t]{5}{*}{$2 b_{1}(\pi)$} & $4 b_{2}(\sigma)$ & $6 b_{2}\left(\sigma^{*}\right)$ & 0.089 & 0.089 & -1.035 & 23.97 \\
\hline & $4 a_{1}(\sigma)$ & $8 a_{1}\left(\sigma^{*}\right)$ & 0.085 & 0.088 & -1.068 & 25.99 \\
\hline & $6 \mathrm{a}_{1}(\sigma)$ & $9 \mathrm{a}_{1}\left(\sigma^{*}\right)$ & 0.060 & 0.061 & 0.854 & 23.82 \\
\hline & $5 a_{1}(\sigma)$ & $0 a_{1}\left(\sigma^{*}\right)$ & 0.053 & 0.056 & -0.860 & 26.50 \\
\hline & $5 b_{2}(\sigma)$ & $7 b_{2}\left(\sigma^{*}\right)$ & 0.049 & 0.050 & 0.758 & 22.91 \\
\hline \multirow[t]{5}{*}{$5 b_{2}(\sigma)$} & $4 b_{2}(\sigma)$ & $6 b_{2}\left(\sigma^{*}\right)$ & 0.064 & 0.065 & -0.882 & 23.97 \\
\hline & $1 a_{2}(\pi)$ & $3 \mathrm{a}_{2}\left(\pi^{*}\right)$ & 0.060 & 0.061 & 0.978 & 31.46 \\
\hline & $4 a_{1}(\sigma)$ & $8 \mathrm{a}_{1}\left(\sigma^{*}\right)$ & 0.058 & 0.055 & -0.847 & 25.99 \\
\hline & $2 b_{1}(\pi)$ & $3 b_{1}\left(\pi^{*}\right)$ & 0.049 & 0.051 & 0.911 & 32.85 \\
\hline & $5 a_{1}(\sigma)$ & $10 \mathrm{a}_{1}\left(\sigma^{*}\right)$ & 0.045 & 0.043 & -0.755 & 26.50 \\
\hline
\end{tabular}


Table IX. The equilibrium geometrical parameters of pyridine (Figure 1) in its electronic ground-state, ${ }^{1} \mathrm{~A}_{1}(\mathrm{GS})$, and the lowest cationic states, ${ }^{2} \mathrm{~A}_{1}\left(7 \mathrm{a}_{1}{ }^{-1}\right),{ }^{2} \mathrm{~A}_{2}\left(1 \mathrm{a}_{2}{ }^{-1}\right)$, and ${ }^{2} \mathrm{~B}_{1}\left(2 \mathrm{~b}_{1}{ }^{-1}\right)$, obtained using the MP2/cc-pVTZ approach (bond lengths in $\AA$, angles in degrees).

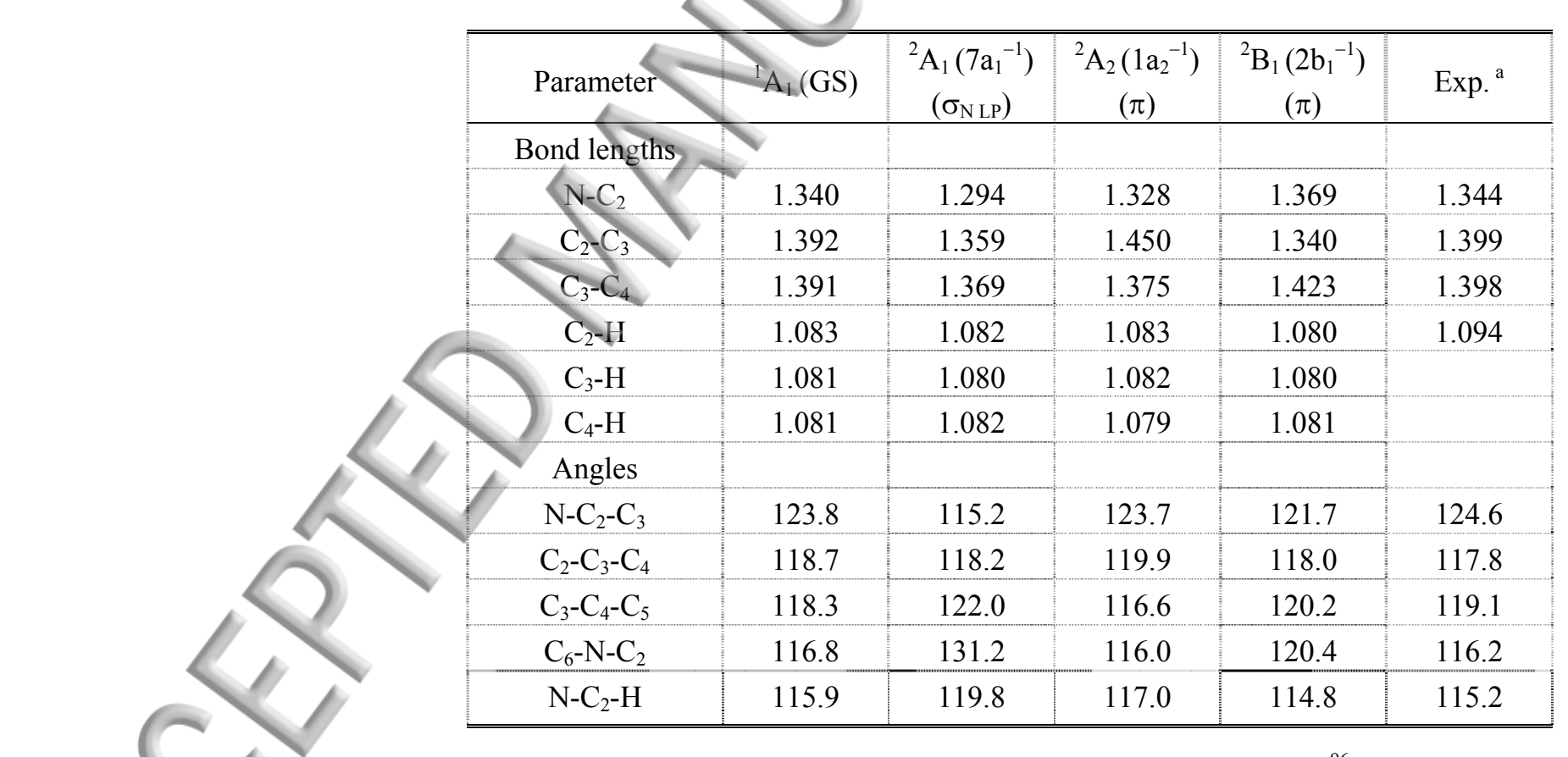

${ }^{a}$ Equilibrium ground-state geometrical parameters of neutral pyridine. ${ }^{96}$ 
Table X. The equilibrium geometrical parameters of pyridine (Figure 1) in its electronic ground-state, ${ }^{1} \mathrm{~A}_{1}(\mathrm{GS})$, and the lowest cationic states, ${ }^{2} \mathrm{~A}_{1}\left(7 \mathrm{a}_{1}{ }^{-1}\right),{ }^{2} \mathrm{~A}_{2}\left(1 \mathrm{a}_{2}{ }^{-1}\right),{ }^{2} \mathrm{~B}_{1}\left(2 \mathrm{~b}_{1}{ }^{-1}\right)$, and ${ }^{2} \mathrm{~B}_{2}\left(5 \mathrm{~b}_{2}{ }^{-1}\right)$, obtained using the CCSD/cc-pVTZ approach (bond lengths in $\AA$, angles in degrees).

\begin{tabular}{|c|c|c|c|c|c|c|c|}
\hline Parameter & ${ }^{1} \mathrm{~A}_{1}(\mathrm{GS})$ & $\begin{array}{l}A_{1}\left(7 a_{1}{ }^{-1}\right) \\
\left(\sigma_{N}{ }^{\prime}\right)\end{array}$ & $\begin{array}{c}{ }^{2} \mathrm{~A}_{2}\left(1 \mathrm{a}_{2}{ }^{-1}\right) \\
(\pi)\end{array}$ & $\begin{array}{c}{ }^{2} \mathrm{~B}_{1}\left(2 \mathrm{~b}_{1}{ }^{-1}\right) \\
(\pi)\end{array}$ & $\begin{array}{c}{ }^{2} \mathrm{~B}_{2}\left(5 \mathrm{~b}_{2}{ }^{-1}\right) \\
(\sigma)\end{array}$ & $\operatorname{Exp}\left({ }^{1} \mathrm{~A}_{1}\right)^{\mathrm{a}}$ & Exp. $\left({ }^{2} \mathrm{~A}_{1}\right)^{\mathrm{b}}$ \\
\hline \multicolumn{8}{|l|}{ Bond lengths } \\
\hline $\mathrm{N}-\mathrm{C}_{2}$ & . & 1.310 & 1.326 & 1.368 & 1.340 & 1.344 & 1.339 \\
\hline $\mathrm{C}_{2}-\mathrm{C}_{3}$ & & 1.393 & 1.451 & 1.367 & 1.392 & 1.399 & 1.392 \\
\hline $\mathrm{C}_{3}-\mathrm{C}_{4}$ & & 1.391 & 1.385 & 1.432 & 1.391 & 1.398 & 1.390 \\
\hline $\mathrm{C}_{2}-\mathrm{H}$ & & 1.081 & 1.083 & 1.080 & 1.083 & 1.094 & 1.082 \\
\hline $\mathrm{C}_{3}-\mathrm{H}$ & 1,080 & 1.080 & 1.081 & 1.080 & 1.081 & & 1.082 \\
\hline $\mathrm{C}_{4}-\mathrm{H}$ & 1.081 & 1.081 & 1.079 & 1.083 & 1.081 & & 1.082 \\
\hline & 123.9 & 114.3 & 124.2 & 122.6 & 123.8 & 124.6 & 114.2 \\
\hline & 118.5 & 118.9 & 119.5 & 117.6 & 118.7 & 117.8 & 119.3 \\
\hline $\mathrm{C}_{3}-\mathrm{C}_{4}-\mathrm{C}_{5}$ & 118.4 & 121.2 & 116.7 & 119.9 & 118.3 & 119.1 & 121.8 \\
\hline $\mathrm{C}_{6}-\mathrm{N}-\mathrm{C}_{2}$ & 116.9 & 132.5 & 116.0 & 119.7 & 116.8 & 116.2 & 131.2 \\
\hline $\mathrm{N}-\mathrm{C}_{2}-\mathrm{H}$ & 115.9 & 119.9 & 116.8 & 115.3 & 115.9 & 115.2 & 119.7 \\
\hline
\end{tabular}

${ }^{a}$ Equilibrium ground-state geometrical parameters of neutral pyridine. ${ }^{96}$

${ }^{\mathrm{b}}$ Geometrical parameters of the pyridine cation-radical obtained from the MATI spectra using a Franck-Condon fitting procedure. ${ }^{39}$ 
Table XI. The harmonic vibrational frequencies $\left(\mathrm{cm}^{-1}\right)$ of pyridine in its electronic ground-state, ${ }^{1} \mathrm{~A}_{1}(\mathrm{GS})$, and the lowest cationic states, ${ }^{2} \mathrm{~A}_{1}\left(7 \mathrm{a}_{1}{ }^{-1}\right),{ }^{2} \mathrm{~A}_{2}\left(1 \mathrm{a}_{2}{ }^{-1}\right)$, and ${ }^{2} \mathrm{~B}_{1}\left(2 \mathrm{~b}_{1}{ }^{-1}\right)$, computed using the MP2/cc-pVTZ approach.

\begin{tabular}{|c|c|c|c|c|c|c|c|}
\hline \multicolumn{2}{|c|}{$\begin{array}{c}\text { Vibrational modes } v \\
\text { and assignment }\end{array}$} & ${ }^{1} \mathrm{~A}_{1}(\mathrm{GS})$ & $\begin{array}{c}{ }^{2} A_{1}\left(7 a_{1}^{-1}\right) \\
\left(\sigma_{N L P}\right)\end{array}$ & $\begin{array}{c}{ }^{2} \mathrm{~A}_{2}\left(1 \mathrm{a}_{2}{ }^{-1}\right) \\
(\pi)\end{array}$ & $\begin{array}{c}{ }^{2} \mathrm{~B}_{1}\left(2 b_{1}^{-1}\right) \\
(\pi)\end{array}$ & $\begin{array}{l}\text { Exp. } \\
\left({ }^{1} \mathrm{~A}_{1}\right)^{\mathrm{b}}\end{array}$ & $\begin{array}{l}\text { Exp. } \\
\left({ }^{2} \mathrm{~A}_{1}\right)^{\mathrm{c}}\end{array}$ \\
\hline \multicolumn{8}{|c|}{$a_{1}$ modes } \\
\hline $6 a$ & $\alpha r$ & 602 & 662 & 587 & 594 & 005 & 604 \\
\hline 1 & $\alpha r$ & 1009 & 980 & 1001 & 882 & 991 & 920 \\
\hline 12 & $\alpha r$ & 1048 & 1135 & 1031 & 1016 & 1030 & 974 \\
\hline $18 \mathrm{a}$ & $\beta \mathrm{CH}$ & 1093 & 1220 & 1197 & 1063 & 1069 & 995 \\
\hline $9 \mathrm{a}$ & $\beta \mathrm{CH}$ & 1242 & 1234 & 1275 & 1266 & 1217 & 1119 \\
\hline $19 a$ & $v r$ & 1508 & 1564 & 1552 & 1427 & 1483 & 1397 \\
\hline $8 \mathrm{a}$ & $v r$ & 1632 & 1832 & 1636 & 1891 & 1581 & 1492 \\
\hline $20 \mathrm{a}$ & $v \mathrm{CH}$ & 3201 & 3240 & 3234 & 3240 & 3025 & \\
\hline 13 & $v \mathrm{CH}$ & 3219 & 3249 & 3243 & 3257 & 3057 & \\
\hline 2 & $v \mathrm{CH}$ & 3244 & 3273 & 3268 & 3274 & 3070 & \\
\hline \multicolumn{8}{|c|}{$\mathrm{b}_{1}$ modes } \\
\hline $16 \mathrm{~b}$ & $\phi \mathrm{r}$ & 414 & 403 & 334 & 326 & 406 & 409 \\
\hline 11 & $\gamma \mathrm{CH}$ & 726 & 647 & 506 & $2266 i$ & 703 & 522 \\
\hline 4 & $\phi r$ & 759 & 879 & 748 & 762 & 747 & \\
\hline $17 \mathrm{~b}$ & $\gamma \mathrm{CH}$ & 953 & 1145 & 958 & 1004 & 941 & \\
\hline 5 & $\gamma \mathrm{CH}$ & 1007 & 1242 & 1096 & 1150 & 1007 & \\
\hline \multicolumn{8}{|c|}{$\mathrm{b}_{2}$ modes } \\
\hline $6 \mathrm{~b}$ & $\alpha r$ & 658 & 617 & 534 & 784 & 654 & 548 \\
\hline $18 \mathrm{~b}$ & $\beta \mathrm{CH}$ & 1079 & 1126 & 982 & $2267 i$ & 1069 & \\
\hline 15 & $\beta \mathrm{CH}$ & 1171 & 1184 & 1148 & 1180 & 1146 & \\
\hline 3 & & 1380 & 1262 & 1353 & 1218 & 1227 & \\
\hline 14 & & 1402 & 1359 & 1406 & 1399 & 1355 & \\
\hline $19 \mathrm{~b}$ & & 1475 & 1602 & 1440 & 1474 & 1437 & \\
\hline $8 \mathrm{~b}$ & $v \mathrm{r}$ & 1622 & 1989 & 1496 & 1650 & 1574 & \\
\hline & $\mathrm{vCH}$ & 3199 & 3244 & 3230 & 3253 & 3034 & \\
\hline & $\mathrm{NCH}$ & 3236 & 3271 & 3245 & 3270 & 3079 & \\
\hline $16 \mathrm{a}$ & $\phi r$ & 382 & 496 & 197 & 338 & 380 & 317 \\
\hline $10 \mathrm{a}$ & $\gamma \mathrm{CH}$ & 906 & 909 & 788 & 934 & 884 & \\
\hline $17 \mathrm{a}$ & $\gamma \mathrm{CH}$ & 998 & 1187 & 1065 & 1151 & 980 & \\
\hline
\end{tabular}

The normal mode is numbered according to Langseth and Lord ${ }^{98}$ and its description is given in terms of the ring $(r)$ and bonds stretching $(v)$, out-of-plane deformations $(\gamma)$, in-plane deformations $(\beta)$, in-plan ring deformation $(\alpha)$, and non-planar ring deformations $(\phi) .{ }^{100}$

${ }^{\mathrm{b}}$ Equilibrium ground-state vibrational frequencies of neutral pyridine. ${ }^{96}$

${ }^{c}$ Vibrational frequencies obtained from the MATI spectra. ${ }^{39}$ 


\section{FIGURE CAPTIONS}

Figure 1.

Schematic representation of pyridine showing the atomic numbering.

Figure 2.

Plots of the $7 a_{1}\left(\sigma_{N L P}\right), 1 a_{2}(\pi), 2 b_{1}(\pi)$, and $5 b_{2}(\sigma)$ Dyson orbitals for ionization of pyridine computed at the ADC(3)/cc-pVDZ level of theory, and the total electron density difference plots $\Delta \rho$ in the respective cationic states and the pyridine ground-state computed at the $\mathrm{CCD} / \mathrm{cc}-\mathrm{pVTZ}$ level of theory for two positions of the plot plane (coinciding with the molecular plane and elevated by $0.5 \AA$ above the molecular plane). The molecular orientation in the contour plots is chosen with $\mathrm{N}$ at the lowest vertex; red and blue contours indicate increase and decrease of density, respectively.

Figure 3.

Contour plots of pyridine total electron density differences showing the effects of orbital relaxation and electron correlation for ionization from the $7 \mathrm{a}_{1}\left(\sigma_{\mathrm{N} L P}\right)$ and $5 \mathrm{~b}_{2}(\sigma)$ orbitals. The differences showing the relaxation effect were produced by subtracting the density obtained in the HF/cc-pVTZ calculations for the ${ }^{1} \mathrm{~A}_{1}$ ground-state from the densities obtained in the $\mathrm{ROH} / \mathrm{cc}-\mathrm{pVTZ}$ calculations for the cationic states. The differences showing electron correlation effects were obtained by subtracting the UHF/cc-pVTZ densities from the densities obtained in the CCD/cc-pVTZ calculations based on the UHF orbitals. The plot plane coincides with the molecular plane with the molecule orientated to place the $\mathrm{N}$ atom at the lawest vertex; the red and blue contours indicate increase and decrease of density, respectively.

Figure 4.

(a) The valence shell photoelectron spectrum of pyridine recorded at a photon energy of 80 $\mathrm{eV}$ and at $\theta=0^{\circ}$. (b) The theoretical photoelectron spectrum of pyridine obtained using the ADC(3) method. 
Figure 5.

The valence shell photoelectron spectrum of pyridine recorded at a photon energy of $40 \mathrm{eV}$ for $\theta=0^{\circ}$ and $\theta=90^{\circ}$.

Figure 6.

The experimental and theoretical (CMS-X $\alpha$ ) photoelectron anisotropy parameters of pyridine associated with the $7 a_{1}\left(\sigma_{N} L P\right), 1 a_{2}(\pi), 2 b_{1}(\pi)$ and $5 b_{2}(\sigma)$ orbitals, corresponding to binding energy regions $1-3$ specified in Table I.

Figure 7.

The experimental and theoretical (CMS-X $\alpha$ ) photoionization partial cross-sections of pyridine associated with the $7 a_{1}\left(\sigma_{N} L P\right), 1 a_{2}(\pi), 2 b_{1}(\pi)$ and $5 b_{2}(\sigma)$ orbitals, corresponding to binding energy regions $1-3$ specified in Táble I. The experimental partial cross-sections have been obtained by combining the measured branching ratios with the absolute photoabsorption cross-section and the photoionization quantum efficiency determined by Tixier et al. ${ }^{93}$ See text for details. 


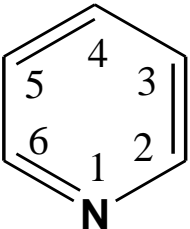




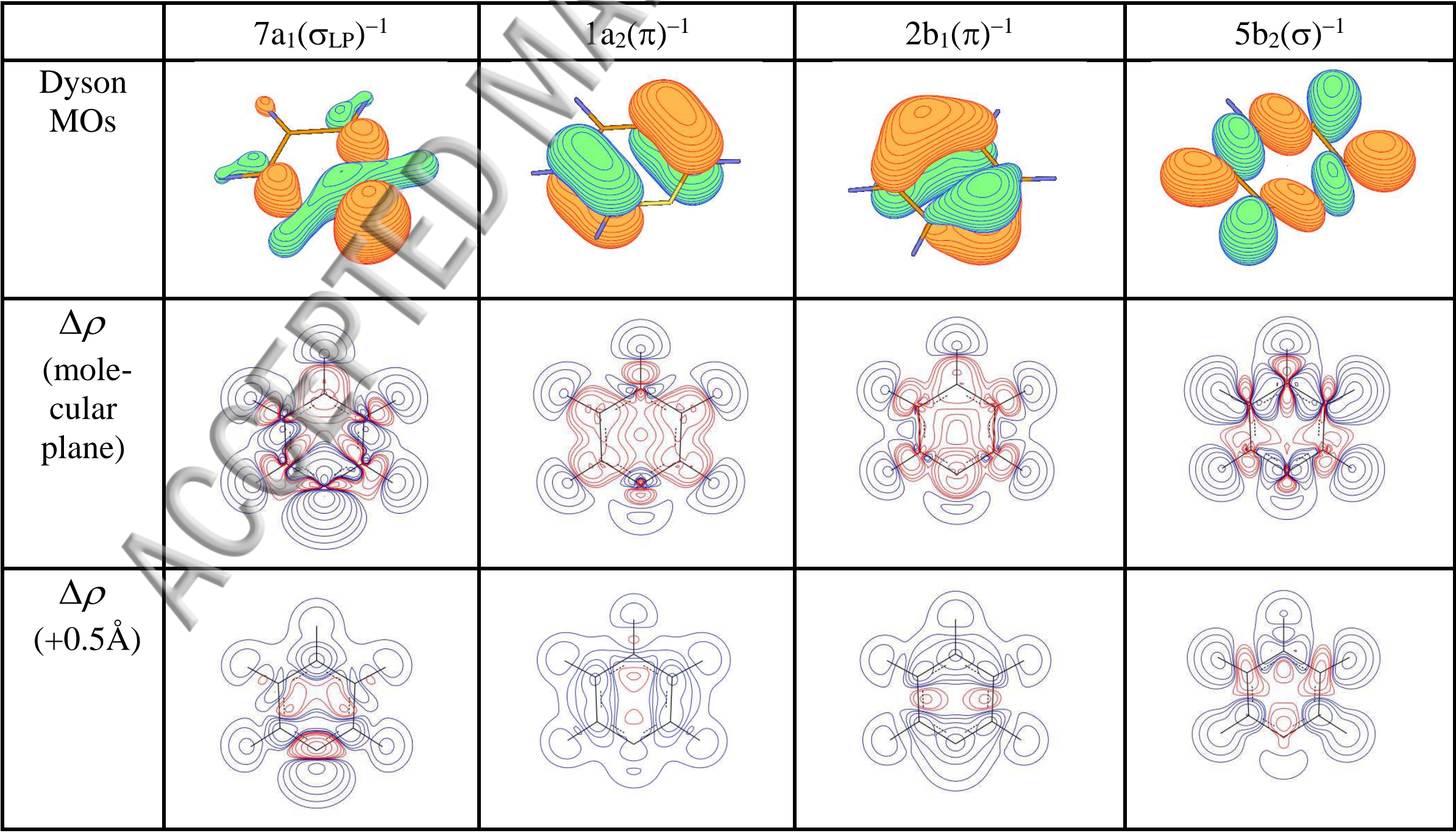









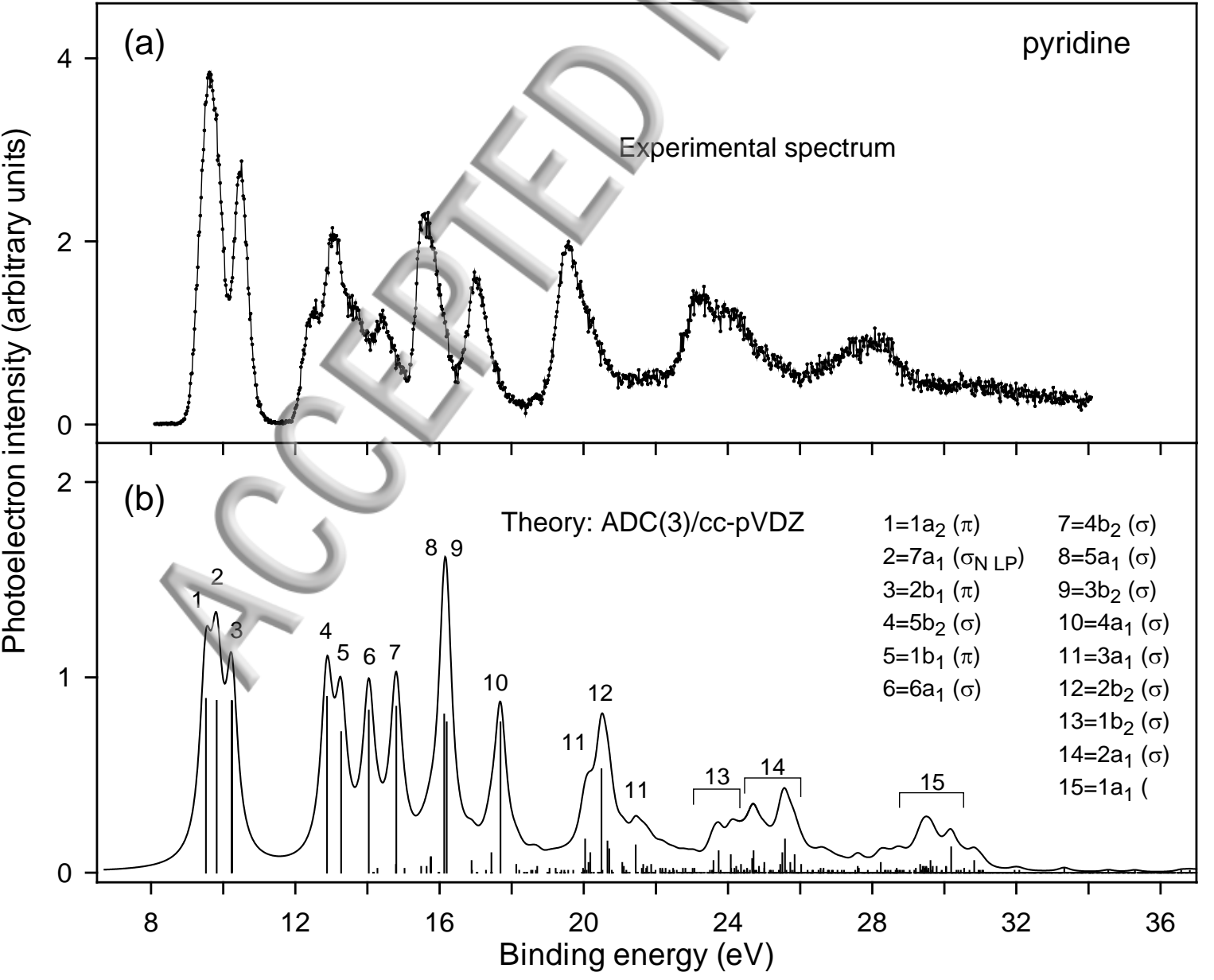




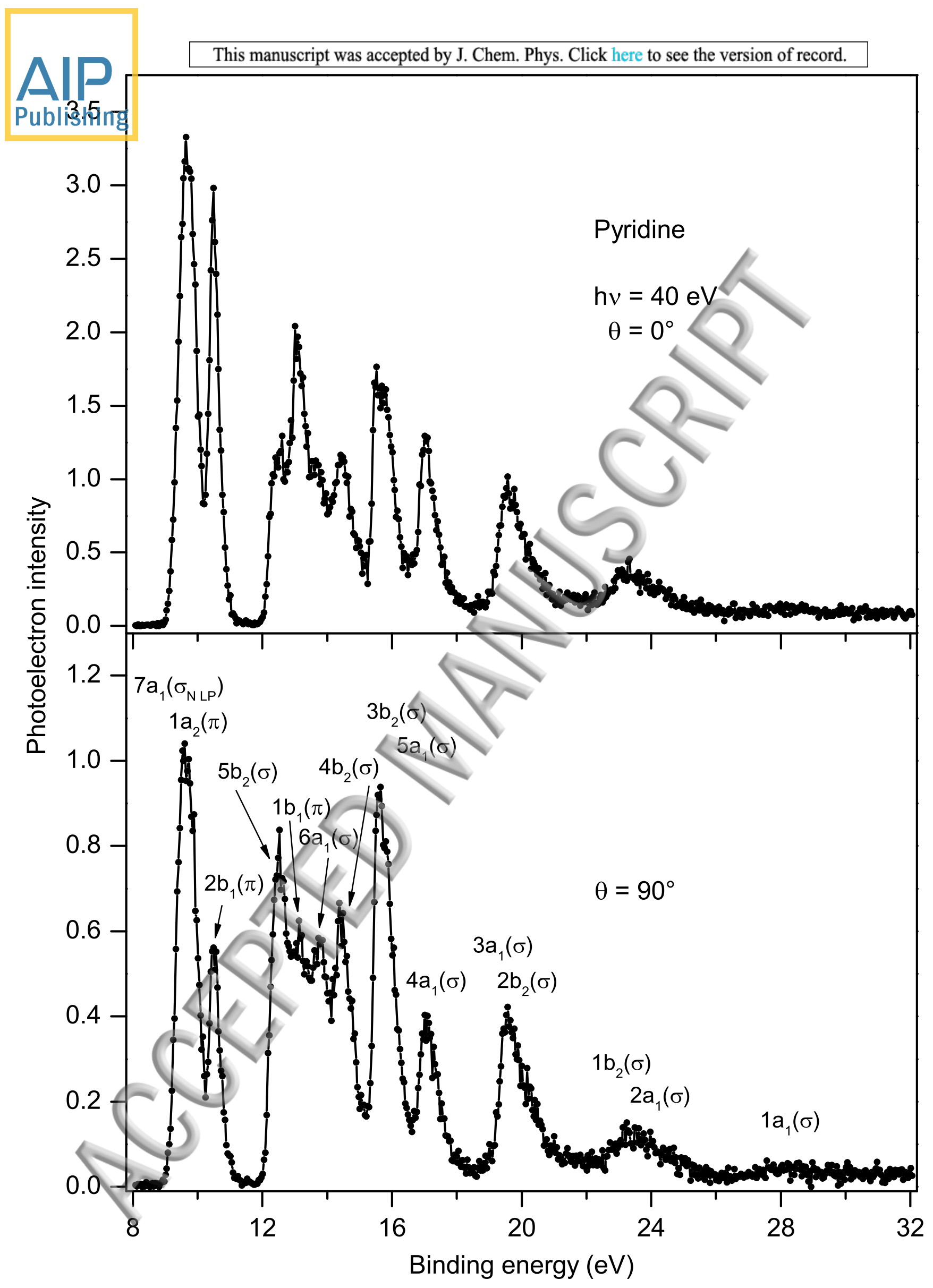




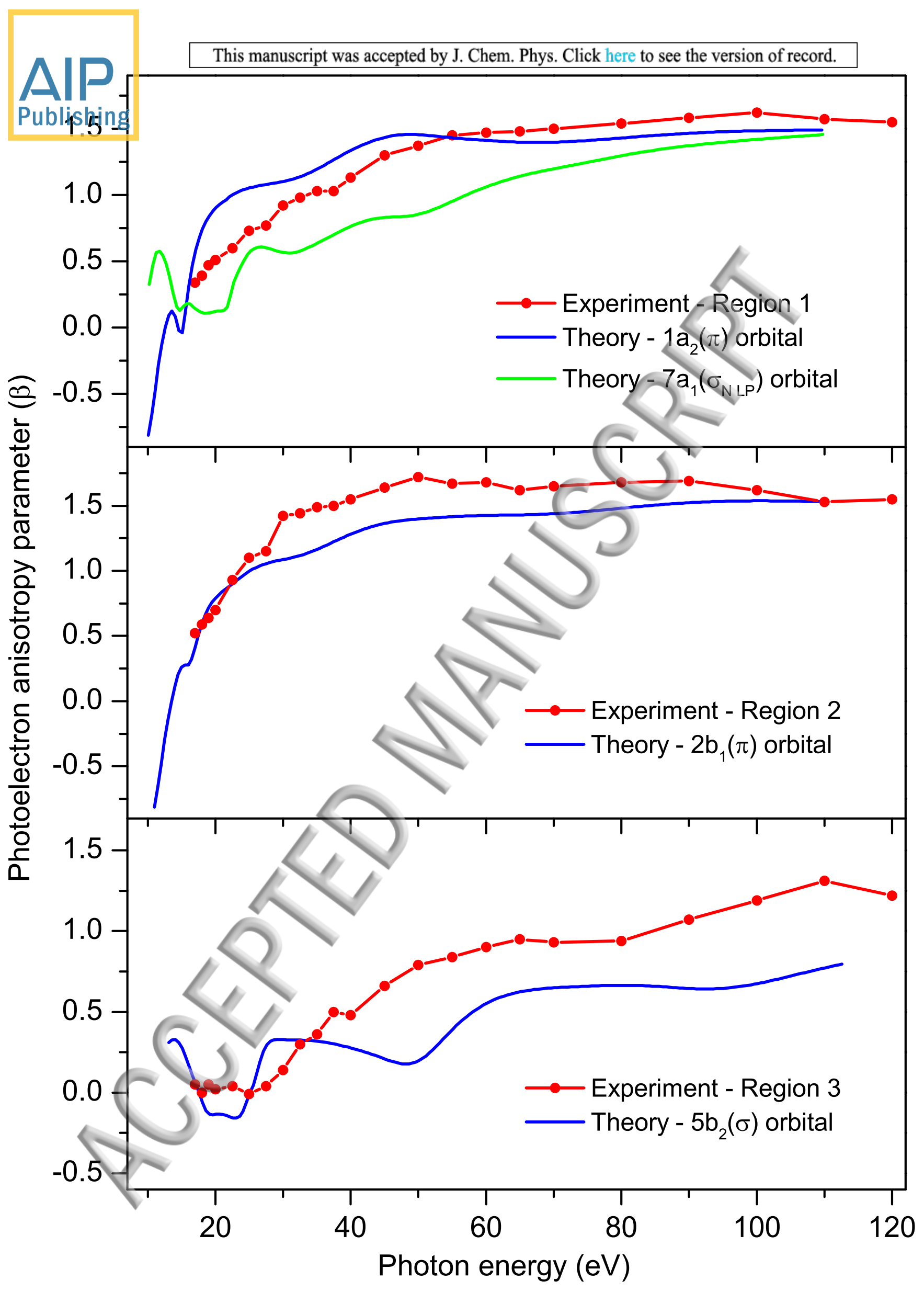




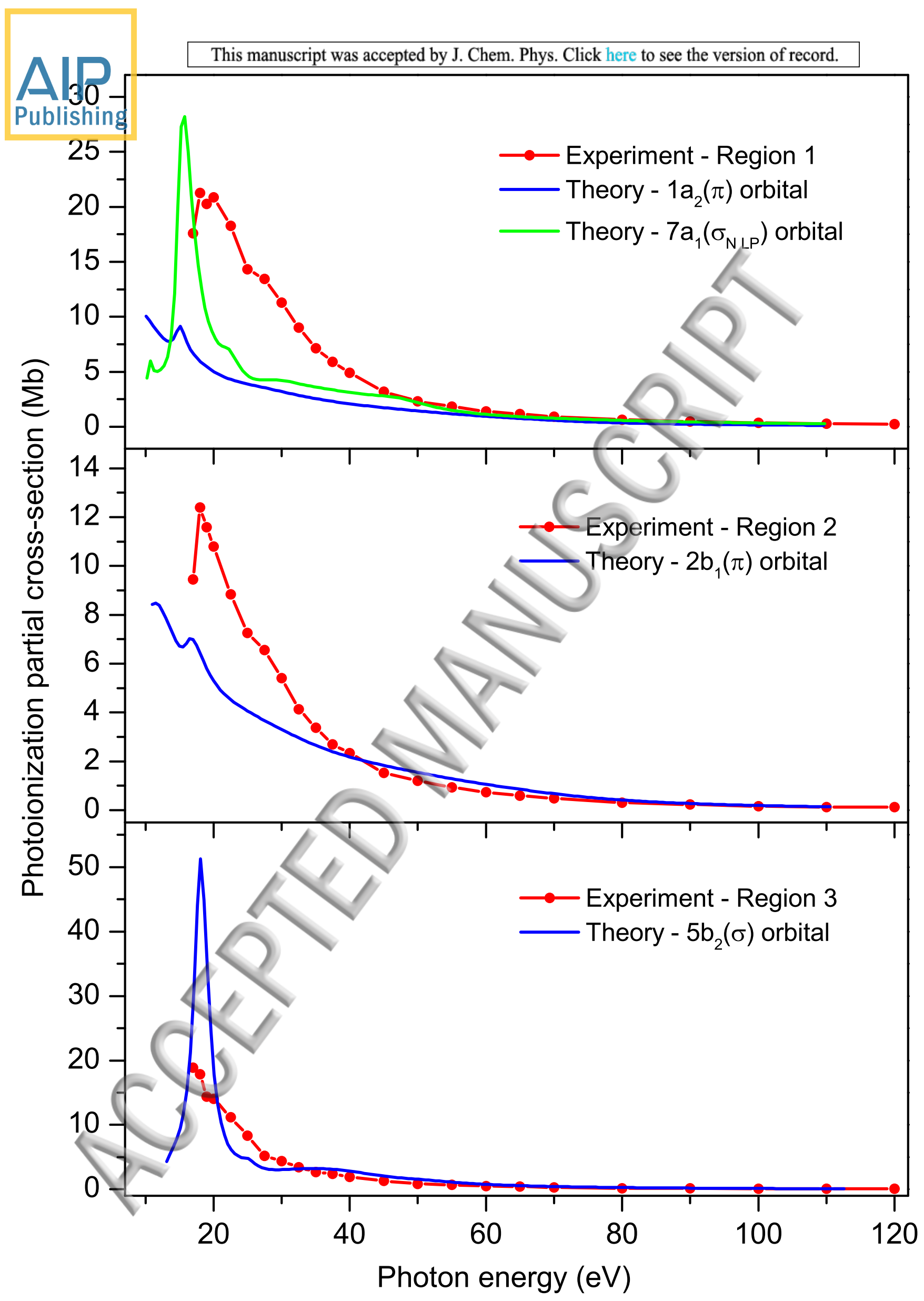

